\title{
Comprehensive modelling of the planetary nebula LMC-SMP 61 and its [WC]-type central star ${ }^{\star}$
}

\author{
G. Stasińska ${ }^{1}$, G. Gräfener ${ }^{2}$, M. Peña ${ }^{3}$, W.-R. Hamann ${ }^{2}$, L. Koesterke ${ }^{4}$, and R. Szczerba ${ }^{5}$ \\ ${ }^{1}$ LUTH, Observatoire de Meudon, 5 place Jules Janssen, 92195 Meudon Cedex, France \\ 2 Institut für Physik, Astrophysik, Universität Potsdam, Am Neuen Palais 10, 14469 Potsdam, Germany \\ e-mail: goetz@astro.physik.uni-potsdam.de \\ 3 Instituto de Astronomía, UNAM, Apdo. Postal 70 264, México D.F., 04510, México \\ e-mail: miriam@astroscu.unam.mx \\ ${ }^{4}$ Laboratory for Astronomy and Solar Physics, NASA Goddard Space Flight Center, Code 681, Greenbelt, MD 20771, USA \\ e-mail: lars@winds.gsfc.nasa.gov \\ 5 N. Copernicus Astronomical Center, Rabiańska 8, 87-100 Toruń, Poland \\ e-mail: szczerba@ncac.torun.pl
}

Received 11 July 2003 / Accepted 18 September 2003

\begin{abstract}
We present a comprehensive study of the Magellanic Cloud planetary nebula SMP 61 and of its nucleus, a Wolf-Rayet type star classified [WC 5-6]. The observational material consists of HST STIS spectroscopy and imaging, together with optical and UV spectroscopic data collected from the literature and infrared fluxes measured by IRAS. We have performed a detailed spectral analysis of the central star, using the Potsdam code for expanding atmospheres in non-LTE. For the central star we determine the following parameters: $L_{\star}=10^{3.96} L_{\odot}, R_{\star}=0.42 R_{\odot}, T_{\star}=87.5 \mathrm{kK}, \dot{M}=10^{-6.12} M_{\odot} \mathrm{yr}^{-1}, v_{\infty}=1400 \mathrm{~km} \mathrm{~s}^{-1}$, and a clumping factor of $D=4$. The elemental abundances by mass are $X_{\mathrm{He}}=0.45, X_{\mathrm{C}}=0.52, X_{\mathrm{N}}<5 \times 10^{-5}, X_{\mathrm{O}}=0.03$, and $X_{\mathrm{Fe}}<1 \times 10^{-4}$. The fluxes from the model stellar atmosphere were used to compute photoionization models of the nebula. All the available observations, within their error bars, were used to constrain these models. We find that the ionizing fluxes predicted by the stellar model are consistent with the fluxes needed by the photoionization model to reproduce the nebular emission, within the error margins. However, there are indications that the stellar model overestimates the number and hardness of Lyman continuum photons. The photoionization models imply a clumped density structure of the nebular material. The observed C III] $\lambda 1909 /$ C II $\lambda 4267$ line ratio implies the existence of carbon-rich clumps in the nebula. Such clumps are likely produced by stellar wind ejecta, possibly mixed with the nebular material. We discuss our results with regard to the stellar and nebular post-AGB evolution. The observed Fe-deficiency for the central star indicates that the material which is now visible on the stellar surface has been exposed to s-process nucleosynthesis during previous thermal pulses. The absence of nitrogen allows us to set an upper limit to the remaining H-envelope mass after a possible AGB final thermal pulse. Finally, we infer from the total amount of carbon detected in the nebula that the strong [WC] mass-loss may have been active only for a limited period during the post-AGB evolution.
\end{abstract}

Key words. stars: Wolf-Rayet - stars: atmospheres - stars: mass-loss - ISM: abundances ISM: planetary nebulae: individual: SMP 61 - ISM: planetary nebulae: general

\section{Introduction}

Only a few studies have been devoted so far to a consistent modelling of a planetary nebula and of its central star. Such studies are useful to get a better insight into the relation between the nebula and its progenitor. Another, very important aspect is that this is the only way to test model atmosphere predictions in the Lyman continuum and thus to validate the model atmospheres. The works of Rauch et al. $(1994,1996)$,

\footnotetext{
Send offprint requests to: G. Stasińska, e-mail: grazyna.stasinska@obspm.fr

$\star$ Table 2 is only available in electronic form at http://www. edpsciences.org
}

Peña et al. (1998), De Marco \& Crowther (1998, 1999), De Marco et al. (2001) are examples of such studies, while Crowther et al. (1999) have performed a similar study on a Population I Wolf-Rayet ring nebula. Such investigations are particularly important in the case of planetary nebulae with Wolf-Rayet type central stars (which represent about $10 \%$ of all planetary nebulae), since recent work (e.g. Górny \& Tylenda 2000; De Marco \& Soker 2002) has completely changed previous views on the evolutionary status of these objects.

In general, Wolf-Rayet central stars of PNe belong to the [WC] sequence. In our galaxy most of these objects have been classified as [WC-early] or [WC-late] types, with only few objects of intermediate types (Tylenda et al. 1993). 
Table 1. Log of observations.

\begin{tabular}{llcrll}
\hline \hline Instrument & Image ID & Date & $\begin{array}{r}\lambda \text { Range } \\
{[\AA]}\end{array}$ & $\begin{array}{r}t_{\exp } \\
{[\mathrm{min}]}\end{array}$ & Remarks \\
\hline CTIO 4 m Ret.2 ${ }^{a}$ & & $31 / 12 / 94$ & $3184-7463$ & 5.0 & 2 and 10" slit width \\
\hline IUE SWP & 54313 & $06 / 04 / 95$ & $1152-1978$ & 135 & Large ap., low res. \\
\hline HST STIS G140L & o57n02010 & $07 / 01 / 99$ & $1124-1730$ & 36.36 & FUV-MAMA \\
HST STIS G140L & o57n02020 & $07 / 01 / 99$ & $1124-1730$ & 22 & FUV-MAMA \\
HST STIS G230L & o57n02030 & $7 / 01 / 99$ & $1580-3160$ & 25.5 & NUV-MAMA \\
HST STIS G230L & o57n02040 & $7 / 01 / 99$ & $1580-3160$ & 54 & NUV-MAMA \\
HST STIS G430L & o57n03010 & $30 / 12 / 98$ & $2900-5710$ & 37.86 & CCD \\
HST STIS image & o57n02iuq & $06 / 01 / 99$ & 5852 & 3.3 & CCD, acquisition im. \\
HST STIS image & o57n03bqq & $30 / 12 / 98$ & 5852 & 3.3 & CCD, acquisition im. \\
\hline
\end{tabular}

${ }^{a}$ CTIO 4 m with Reticon detector and grating KPGL2.

Also in HST archives FOS spectra Y2N30302T, Y2N30303T, Y2N30304T, 1150-4800 A reported by Vassiliadis et al. (1998b).

In the Magellanic Clouds, the WR central stars are also of [WC] type (except for the extraordinary central star of LMCN66, e.g. Peña et al. 1997b), but in this case they have been classified in the intermediate [WC] types. Peña et al. (1997a) suggested that such a difference might be a consequence of the differences in metallicity between the Milky Way and the Magellanic Clouds. In any case, [WC] central stars show spectral features identical to those of massive WC stars but at much lower luminosity, and they can be analyzed with the tools developed for massive WR stars (e.g. Hamann 1997).

In the present paper, we concentrate on the planetary nebula SMP 61 (also known as N203, WS 24 and LM1-37) which is among the brightest planetary nebulae in the Large Magellanic Cloud. This is a good case for a detailed study: The object is at a known distance modulus of 18.50 mag (Benedict et al. 2002), equivalent to $50.1 \mathrm{kpc}$. The central star is one of the brightest [WC] central stars in the LMC and therefore relatively easy to observe. It is of [WC 5-6] type (Monk et al. 1988; Peña et al. 1997a), therefore the nebular spectrum contains many lines of various excitation levels that allow refined diagnostics. In addition, the nebula appears to be spherical and integrated spectra are already available (Peña et al. 1997a).

Using HST STIS, we have secured high signal-to-noise spectra of SMP61 in a wide spectral range. This provided strong constraints for our modelling of the central star atmosphere. The best fit model atmosphere was then used as an input to build a photoionization model for the nebula. The observations are described in Sect. 2, the stellar modelling technique is presented in Sect. 3, the spectral analysis of the central star of SMP 61 is discussed in Sect. 4. The nebular modelling strategy is exposed in Sect. 5 and the nebular model fitting of SMP 61 is presented in Sect. 6. The implications of our modelling are discussed in Sect. 7, and the main points of this study are summarized in Sect. 8.

\section{New observations and previous data}

\subsection{Observations}

HST STIS spectroscopic data were obtained on 1998/12/30 (HST Cycle 7, program ID 7303). MAMA and CCD detectors were employed to cover the broadest spectral range possible. Prior to the spectroscopic observations, STIS images were obtained to acquire the target. The log of the observations is presented in Table 1.

Direct images with HST WFPC were searched in the archives. Apart from our own STIS images for target acquisition (see Table 1), we retrieved the FOC image W1ID0601T, obtained on 1993/11/08, with exposure time of $3 \mathrm{~min}$ through the filter F502N which isolated the strong [O III] $\lambda 5007$ line (this image is shown in Vassiliadis et al. 1998b). We decided to use our target acquisition images to analyze the nebular morphology and surface brightness because they are better exposed and because the Vassiliadis et al. images were obtained before the COSTAR mission. Our images are obtained with a filter including the brightest emission lines: $\mathrm{H} \alpha, \mathrm{H} \beta$ and [O III] lines.

We also considered ground-based spectrophotometric data for SMP 61 obtained by Monk et al. (1988), Meatheringham \& Dopita (1991) and Peña et al. (1997a). Other available UV spectroscopic data are IUE data given in Peña et al. (1997a) and HST FOS data from 1175 to $4800 \AA$ published by Vassiliadis et al. (1998a).

All the available spectroscopic data are presented in Table 2, which lists the reddening corrected fluxes for the observed lines (in units of $\mathrm{H} \beta=100$ ) obtained by the different authors. We also list the total $\mathrm{H} \beta$ flux observed in the slit, $F(\mathrm{H} \beta)$, the logarithmic extinction at $\mathrm{H} \beta, C(\mathrm{H} \beta)$, and the slit dimensions, when available. All the spectra mentioned above have good signal-to-noise, and the differences among observations by different authors are, in general, small. Note that observations by Peña et al. (1997a) were made both with a slit of $2^{\prime \prime}$ and a slit of $10^{\prime \prime}$ and, as noted by these authors, the line ratios are very similar in both slits. The $\mathrm{H} \beta$ flux reported in Col. 2 of Table 2 is the one obtained with the $10^{\prime \prime}$ slit.

SMP 61 is one of the twelve planetary nebulae in the LMC detected by IRAS. The fluxes retrieved from the IRAS data base are: $F(12 \mu \mathrm{m})=0.08 \mathrm{Jy}$ (flux density quality $=2$ ) $F(25 \mu \mathrm{m})=0.13 \mathrm{Jy}$ (flux density quality $=3), F(60 \mu \mathrm{m}) \leq$ $0.16 \mathrm{Jy}$ (flux density quality $=1$ ).

\subsection{Preliminary analysis of the observational data}

In HST images the nebula appears perfectly spherical and shows no small-scale structure. The surface flux distribution, 


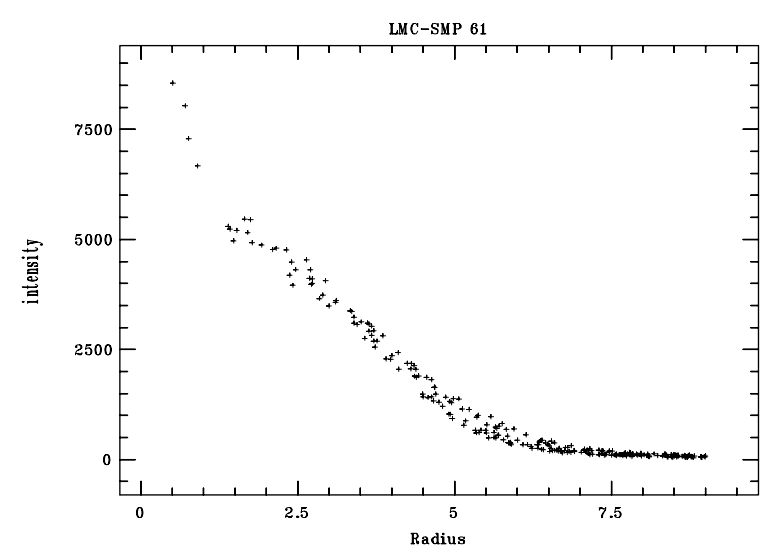

Fig. 1. Radial distribution of the surface brightness. The STIS image 57n02iuq, obtained for target acquisition, was employed to prepare this graphic. The radius is measured in pixels. Each pixel is equivalent to $0.05^{\prime \prime}$.

as derived from the image o57n02iuq, is shown in Fig. 1. The ionizing star dominates the emission in the central pixels. The nebula seems to have an inner radius of about $0.065^{\prime \prime}$, showing the maximum surface brightness there. The surface brightness declines outwards and drops below $1 / 10$ of its maximum at $0.26^{\prime \prime}$.

The extinction of the nebula is modest. Throughout the paper, we use the value $C(\mathrm{H} \beta)=0.18$ (corresponding to $E_{B-V}=$ 0.122 mag derived by Peña et al. (1997a) from the observed Balmer decrement and we adopt the Seaton (1979) reddening law.

The STIS observations were essentially obtained to provide constraints on the central star. However, the $0.2^{\prime \prime} \times 52^{\prime \prime}$ STIS slit includes a significant fraction of the nebula so that both stellar and nebular emission is seen. The nebular emission must of course be subtracted from the stellar emission in order to perform the stellar analysis (see Sect. 4.1). Most of the emission lines detected in the UV are of stellar origin, except for the intense C III] $1909 \AA$ A, C II 2321, $26 \AA$, and [O II] $2470 \AA$. Many bright nebular lines were detected in the optical region. The dereddened fluxes of all the nebular lines measured in the STIS calibrated spectra are presented in the first column of Table 2.

The STIS nebular lines together with the data presented by Peña et al. (1997a, Col. 2 in Table 2) have been used to derive first estimates of the physical conditions and of the chemical composition of the ionized gas. The atomic data are the same as used in the nebular modelling (see Sect. 5). The density derived from [S II] $\lambda 6731 / 6717$ is $5400 \mathrm{~cm}^{-3}$, the temperature derived from [O III] $\lambda 4363 / 5007$ is $10300 \mathrm{~K}$, and the one derived from [N II] $\lambda 5755 / 6584$ is $11400 \mathrm{~K}$. These values were used to derive the ionic abundances, and the ionization correction factors from Kingsburgh \& Barlow (1994) led to the following total abundance ratios: $\mathrm{H}: \mathrm{He}: \mathrm{C}: \mathrm{N}: \mathrm{O}: \mathrm{Ne}: \mathrm{S}: \mathrm{Ar}=1: .104$ : $7.35 \times 10^{-4}: 3.95 \times 10^{-5}: 2.65 \times 10^{-4}: 4.26 \times 10^{-5}: 6.0 \times 10^{-6}$ : $8.0 \times 10^{-7}$. These abundances are employed in Sect. 6 as starting values for the photoionization modelling.

Regarding the IRAS data, Zijlstra et al. (1994) commented that the IRAS colors $F(12) / F(25) \sim 0.62$ and $F(25) / F(60) \leq$ 0.81 of SMP 61 are very blue and typical of a young nebula.

\section{Stellar modelling technique}

\subsection{Model atmospheres}

The analysis of hot stars with strong winds like the [WC]-type central star of SMP 61 requires the application of model atmospheres that account for complex model atoms in non-LTE. Recent investigations revealed the necessity to include iron line-blanketing as well as the effect of density inhomogeneities (clumping) for a reliable determination of their stellar parameters and emergent flux distributions (Hamann \& Koesterke 1998; Hillier \& Miller 1999; Crowther et al. 2002; Gräfener et al. 2002).

In the present work, we utilize the Potsdam code for expanding atmospheres in non-LTE (Koesterke et al. 1992; Hamann et al. 1992; Leuenhagen \& Hamann 1994; Koesterke \& Hamann 1995; Leuenhagen et al. 1996; Hamann \& Koesterke 1998; Koesterke et al. 2002; Gräfener et al. 2002). This code calculates the radiative transfer in the co-moving frame of reference for a spherically symmetric, stationary outflow. The atomic populations, the electron density, and the electron temperature throughout the extended atmosphere are determined from the equations of statistical- and radiative equilibrium. The system of statistical equations is solved in line with the radiation transport by application of the ALI formalism (accelerated lambda iteration, see Hamann 1985, 1986), whereas radiative equilibrium is obtained by a temperature correction procedure which is based on the method of Unsöld (1955) and Lucy (1964) (see Hamann \& Gräfener 2003; Mihalas 1978, p. 174). The statistical equations are solved for complex model atoms of $\mathrm{He}, \mathrm{C}, \mathrm{O}, \mathrm{Si}$, and the iron group, taking advantage of the concept of super levels for the inclusion of millions of iron-group line transitions (see Gräfener et al. 2002). Density inhomogeneities are accounted for by the assumption of small-scale clumps with a constant volume filling factor $f_{\mathrm{V}}$ (Hamann \& Koesterke 1998).

\subsection{Model parameters}

The model atmospheres are specified by the luminosity and radius of the stellar core, by the chemical composition of the envelope, and its density- and velocity structure. The basic parameters are: The stellar core radius $R_{\star}$ at Rosseland optical depth $\tau_{\mathrm{R}}=20$, the stellar temperature $T_{\star}$ (related to the luminosity $L_{\star}$ via the Stefan Boltzmann law), the chemical composition (given by mass fractions $X_{\mathrm{He}}, X_{\mathrm{C}}, X_{\mathrm{O}}, X_{\mathrm{Si}}$, and $X_{\mathrm{Fe}}$ ), the mass-loss rate $\dot{M}$, the terminal velocity $v_{\infty}$, and the "clumping factor" $D=1 / f_{\mathrm{V}}$ giving the density enhancement of the clumped matter with respect to the mean wind density. For the dependence of the velocity on radius, a relation of the form $v(r)=v_{\infty}\left(1-R_{0} / r\right)$ is assumed. This outer velocity law is augmented by an exponentially decreasing density distribution in the hydrostatic domain. $R_{0}$ is suitably determined to connect both domains smoothly.

The parameters $R_{\star}, \dot{M}, v_{\infty}$, and $D$ are connected by the "transformed radius" $R_{\mathrm{t}} \propto R_{\star}\left(v_{\infty} / \sqrt{D} \dot{M}\right)^{2 / 3}$. Models with the same $R_{\mathrm{t}}$ show almost identical line equivalent widths (Schmutz et al. 1989). This invariance holds because the line 


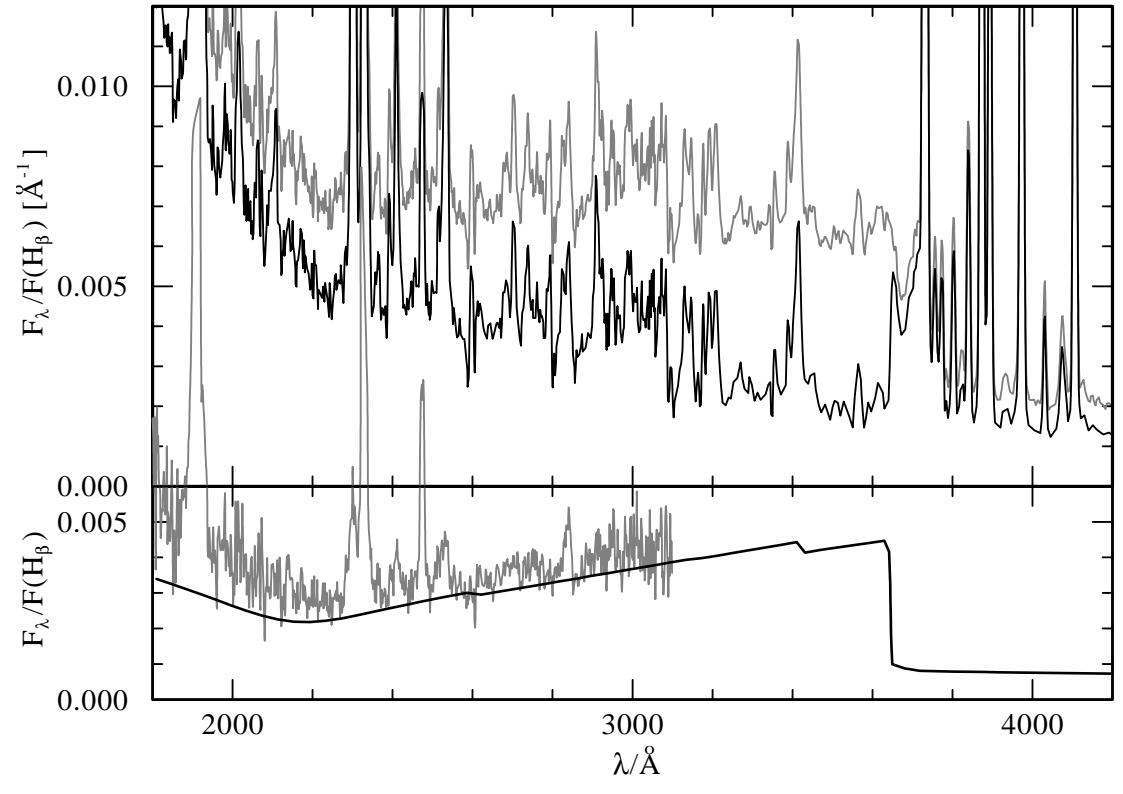

Fig. 2. Subtraction of the nebular continuum. Top panel: The observed flux distribution around the Balmer jump before and after subtraction of the nebular continuum. Bottom panel: The reddened theoretical continuum as utilized for the nebular subtraction is plotted in black. It is compared to the nebular flux (grey) as estimated from the observation, by correcting for the stellar contribution (see text). Although the latter may still include a small contribution from the central star, the theoretical flux distribution is clearly confirmed. emission in WR stars is dominated by recombination processes (see Hamann \& Koesterke 1998).

\section{Spectral analysis of the central star}

In the present section, we perform a detailed spectral analysis of the central star of SMP 61 based on HST UV + optical observations. Owing to the reliable spectrophotometry of HST in combination with the known distance and the moderate interstellar reddening towards the LMC, the observations provide the absolute stellar flux distribution in the wavelength range from 1150 to $6000 \AA$. The model comparisons in the present section are therefore performed with respect to the absolute flux, corrected for interstellar extinction applying the standard law of Seaton (1979) with $E_{B-V}=0.122$ mag.

\subsection{Subtraction of the nebular continuum}

Due to the small angular diameter of the nebula $\left(0.7^{\prime \prime}\right)$, the HST spectra (0.2" slit width) include a significant part of the total nebular flux. Consequently, the observations clearly show nebular features superposed to the stellar spectrum. The most striking of these is the Balmer jump at $3646 \AA$ in emission, indicating the dominance of the nebular continuum in the UV to blue part of the observation. A reliable subtraction of this continuum emission is essential for the spectral analysis of the central star.

A distinction between both contributions is in principle possible, because the nebula is spatially extended in contrast to the point-like star. To estimate the contribution of the nebula, two spectra with apertures of $1.2^{\prime \prime}$ and $0.25^{\prime \prime}$ are extracted from the UV part of the HST data. The difference between these two spectra is dominated by nebular emission. After scaling the difference spectrum to the flux of the nebular C II line at $2327 \AA$, a coarse estimate for the pure nebular flux is obtained.

In Fig. 2, this spectrum is compared to the theoretical nebular continuum flux computed with the physical conditions relevant to the object. After applying the appropriate reddening the model flux is scaled by the ratio of observed to theoretical $\mathrm{H}_{\beta}$ line flux. Finally, the theoretical continuum is utilized for the nebular subtraction. Due to the good agreement between theory and observation, a possible stellar contribution to the observed Balmer jump can be excluded.

\subsection{Spectral fit}

The central star of SMP 61 shows a typical [WC 5]-type spectrum dominated by emission lines of $\mathrm{He}, \mathrm{C}$, and $\mathrm{O}$. Apart from a narrower line width, the spectral appearance of SMP 61 is similar to the well-known massive WC 5 star WR 111. Consequently, the fit criteria and the fitting technique are similar to the analysis of WR 111 described by Gräfener et al. (2002). An important difference between both objects concerns the low iron abundance of SMP 61. The iron forest around $1300 \AA$ - a combination of a large number of iron emission and absorption lines - is barely visible in the HST observations of this object.

The stellar analysis is based on fitting the absolute flux distribution including the observed wind emission lines of He II, C III, C IV, O III, O IV, and OV. A hydrogen-free WC surface composition with a metallicity of 0.3 solar is adopted (referring to the compilation of solar element abundances in Gray 1992, p. 318). This value matches the derived nebular oxygen abundance. The iron mass fraction is correspondingly set to $X_{\mathrm{Fe}}=4 \times 10^{-4}$, and the silicon abundance to $X_{\mathrm{Si}}=2 \times 10^{-4}$. The other model parameters are kept free throughout the fitting procedure. The hydrogen abundance is set to zero. This assumption cannot be checked empirically, because the strong nebular Balmer emission masks any possible stellar contribution. However, in previous works no hydrogen is found on the surface of early-type [WC] stars, and even for late types only small amounts of $X_{\mathrm{H}}<0.1$ are detected (Koesterke 2001; De Marco \& Barlow 2001). 

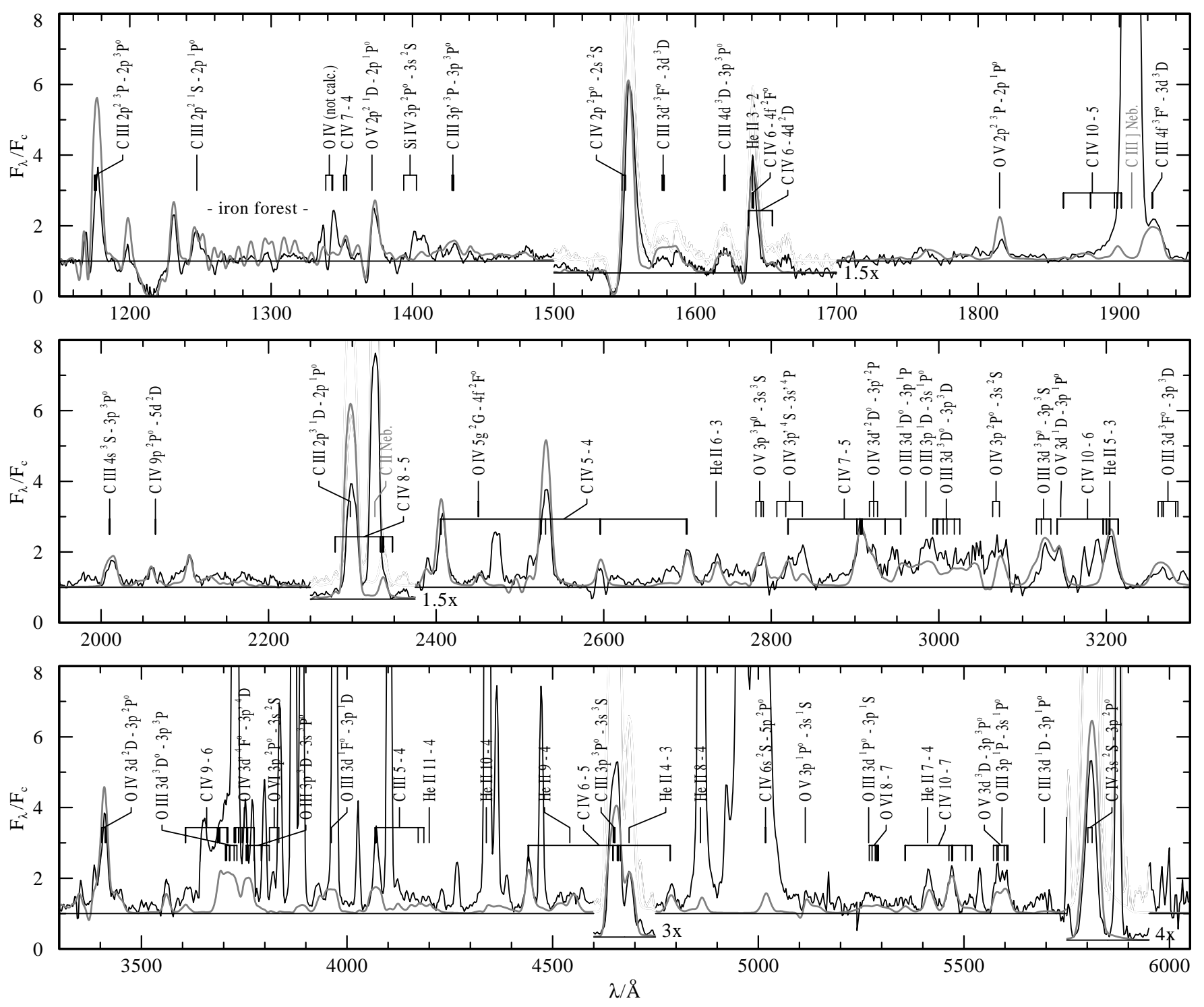

Fig. 3. Spectral fit of the central star of SMP 61. The observation (thin line) is shown together with the synthetic spectrum (thick line, grey). The model parameters are compiled in Table 3. Prominent spectral lines are identified. After correction for interstellar extinction, the observed and the model flux are both divided by the same model continuum, i.e. absolute fluxes are compared. Additionally, a correction for interstellar Ly $\alpha$ absorption is applied to the model spectrum.

The final model fit is presented in Fig. 3 together with the derived stellar parameters in Table 3. As expected, the stellar temperature, transformed radius, and surface composition are similar to the values obtained for WR 111 (Gräfener et al. 2002), while the luminosity is lower by 1.5 dex. A good fit quality is obtained with some exceptions like C III $2297 \AA$ (a transition that is mainly fed by dielectronic recombination), the classification line C III $5696 \AA$, or the $\mathrm{He}$ II/C IV blend at 5412/5470 $\AA$. The latter reacts very sensitively to changes of the ratio of carbon to helium abundance. Additionally, our model with standard iron abundance produces too strong iron features in the UV.

Test calculations for different iron abundances are presented in Fig. 4. For a mass fraction around $5 \times 10^{-5}$ $(\approx 0.04$ solar) the overall strength of the iron forest is well reproduced, but the wavelengths of the single transitions still do not match the observation. For this reason we can only set an upper limit of $X_{\mathrm{Fe}}<1 \times 10^{-4}(\approx 0.07$ solar $)$ to the iron abundance. Compared to the standard LMC metallicity found in the nebula the central star shows a significant iron deficiency. In addition, the absence of N IV line emission at $1721 \AA$, which is relatively common among galactic [WC] stars, allows for the determination of an upper limit for the nitrogen surface abundance. Calculations with a full nitrogen model atom imply a value of $X_{\mathrm{N}}<5 \times 10^{-5}$.

For the subsequent nebular analysis, it is important to discuss the reliability of the derived ionizing fluxes. The main difficulty in the present analysis arises from the nebular continuum dominating the observed flux distribution from $\approx 2500 \AA$ to the visible wavelength range. At first sight, the situation seems to be well established, because the subtraction of the nebular continuum works without difficulties. In addition, 
Table 3. Model parameters for the central star of SMP61. Stellar core parameters: Stellar luminosity $L_{\star}$, temperature $T_{\star}$ and radius $R_{\star}$. Wind parameters: mass-loss rate $\dot{M}$, terminal wind velocity $v_{\infty}$, clumping factor $D$, and the corresponding transformed radius $R_{\mathrm{t}}$. Atmospheric abundances: Mass fractions $X_{\mathrm{He}}, X_{\mathrm{C}}$, and $X_{\mathrm{O}}$ are determined by the model fit (Fig. 3) whereas $X_{\mathrm{Si}}$ is set to a standard LMC value. For $X_{\mathrm{Fe}}$ and $X_{\mathrm{N}}$ upper limits are given. The hydrogen mass fraction $X_{\mathrm{H}}$ could not be determined in the present analysis. Interstellar parameters: Distance modulus $M-m$ for the LMC, color excess due to interstellar extinction $E_{B-V}$, and Ly $\alpha$ hydrogen column density $n_{\mathrm{H}}$.

\begin{tabular}{llll}
\hline \hline$L_{\star}$ & $10^{3.96} L_{\odot}$ & $R_{\star}$ & $0.42 R_{\odot}$ \\
$T_{\star}$ & $87.5 \mathrm{kK}$ & & \\
\hline$\dot{M}$ & $10^{-6.12} M_{\odot} \mathrm{yr}^{-1}$ & $v_{\infty}$ & $1400 \mathrm{~km} \mathrm{~s}^{-1}$ \\
$D$ & 4 & $R_{\mathrm{t}}$ & $4.63 R_{\odot}$ \\
\hline$X_{\mathrm{H}}$ & - & $X_{\mathrm{He}}$ & 0.45 \\
$X_{\mathrm{C}}$ & 0.52 & $X_{\mathrm{N}}$ & $<5 \times 10^{-5}$ \\
$X_{\mathrm{O}}$ & 0.03 & $X_{\mathrm{Si}}$ & $\left(2 \times 10^{-4}\right)$ \\
$X_{\mathrm{Fe}}$ & $<1 \times 10^{-4}$ & & \\
\hline$M-m$ & $18.5 \mathrm{mag}$ & $n_{\mathrm{H}}$ & $1 \times 10^{21} \mathrm{~cm}^{-2}$ \\
$E_{B-V}$ & $0.122 \mathrm{mag}$ & & \\
\hline
\end{tabular}

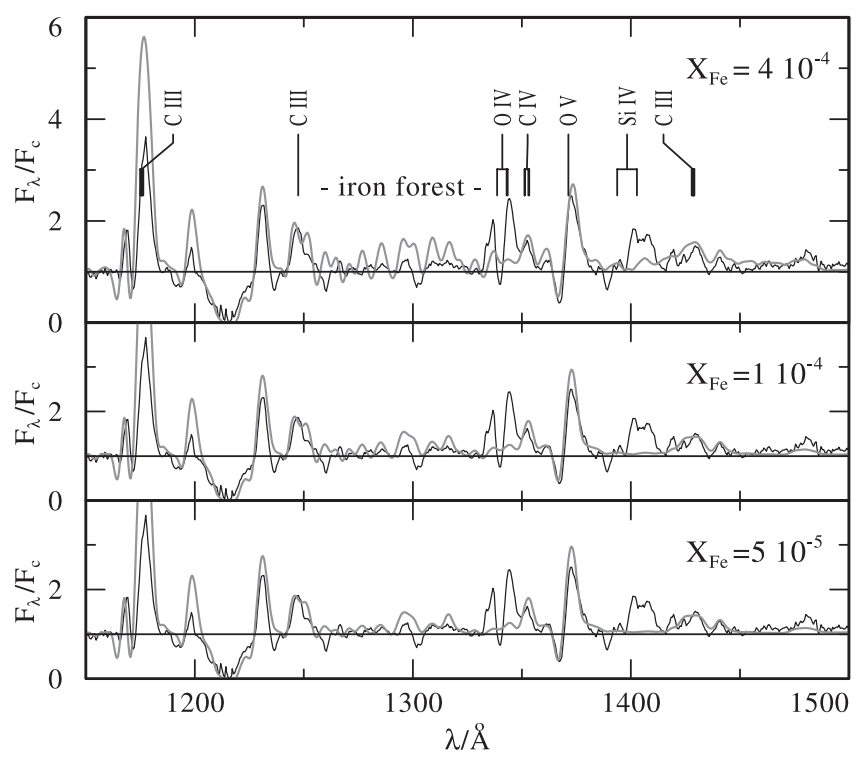

Fig. 4. Model calculations for different iron abundances. The strength of the iron forest indicates a significant iron deficiency on the central stars surface $\left(X_{\mathrm{Fe}}<1 \times 10^{-4}\right)$.

the resulting stellar flux distribution is very well reproduced by our models, and the extinction parameter determined from the nebular spectrum is perfectly compensating the interstellar $2200 \AA$ feature (see Fig. 5). However, slight uncertainties of the flux level in the optical wavelength range remain. For this reason, the determination of the stellar luminosity primarily relies on the UV, i.e. it becomes extremely sensitive to the applied reddening parameter $E_{B-V}$.

Moreover, already small changes of the model parameters may significantly affect the derived values for the stellar luminosity and ionizing fluxes, because the observed flux distribution is still far away from the flux maximum at $\approx 350 \AA$. Test calculations indicate that a variation of the model parameters, leading towards an inferior but still acceptable fit quality, can change the derived luminosity up to $0.1-0.2$ dex. Including the observational uncertainties mentioned above, the possible error may reach values up to 0.3 dex.

In Fig. 5, the overall flux distribution as obtained from our model calculations is shown together with the observed flux distribution, and the ionization edges of O II, He I and H I. The importance of testing the ionizing fluxes for a reliable determination of the overall flux distribution of the central star is obvious. A detailed analysis of the nebular spectrum is therefore capable to put substantial constraints on our atmosphere calculations.

\section{Nebular modelling technique}

\subsection{The photoionization code}

The photoionization models for the nebula were constructed using the code PHOTO which computes the radiative transfer of the ionizing photons in spherical symmetry with the "outward only" assumption for the diffuse radiation field and solves at each radius the equations of ionization equilibrium and thermal balance. The atomic data are the same as used by Stasińska \& Leitherer (1996). The only modification is that low-temperature dielectronic recombination coefficients for $\mathrm{S}$ and Ar ions were introduced (assuming that they are equal to the computed values for the corresponding second-row elements, $\mathrm{O}$ and $\mathrm{Ne}$ respectively). The effects of dust are treated as described in Stasińska \& Szczerba (1999, 2001).

\subsection{The strategy}

The aim is to reproduce all the observational constraints within a certain degree of tolerance (to be clarified later). The observational constraints are the emission line fluxes in the optical and the ultraviolet, the fluxes in the $12 \mu \mathrm{m}, 25 \mu \mathrm{m}$ and $60 \mu \mathrm{m}$ IRAS bands and the surface brightness distribution as measured by HST. Out of the zone affected by the central star, the surface brightness distribution is dominated by the [O III] $\lambda 4959,5007, \mathrm{H} \beta$ and $\mathrm{H} \alpha$ lines (with the [O III] lines giving the major contribution except, perhaps, in the outer parts of the nebula).

The models are computed using as an input the radiation field from the stellar atmospheres described in the previous section. The other input parameters are the inner nebular radius, the gas density distribution, the abundances of the elements and the characteristics of the dust grain population.

We assume that dust is composed of graphite grains with a classical power-law size distribution of exponent -3.5 between $0.003 \mu \mathrm{m}$ and $0.3 \mu \mathrm{m}$. The total dust-to-gas mass ratio is chosen so as to reproduce the observed flux in the $25 \mu \mathrm{m}$ IRAS band (which includes nebular fine structure lines). As a matter of fact, in SMP 61, the observed IRAS fluxes correspond to a small amount of dust, which then does not affect significantly the nebular $\mathrm{H} \beta$ luminosity or its ionization structure. We do not introduce an additional population of small organic grains, as in Stasińska \& Szczerba (2001), since the effects of those grains are essentially to heat the gas and, 


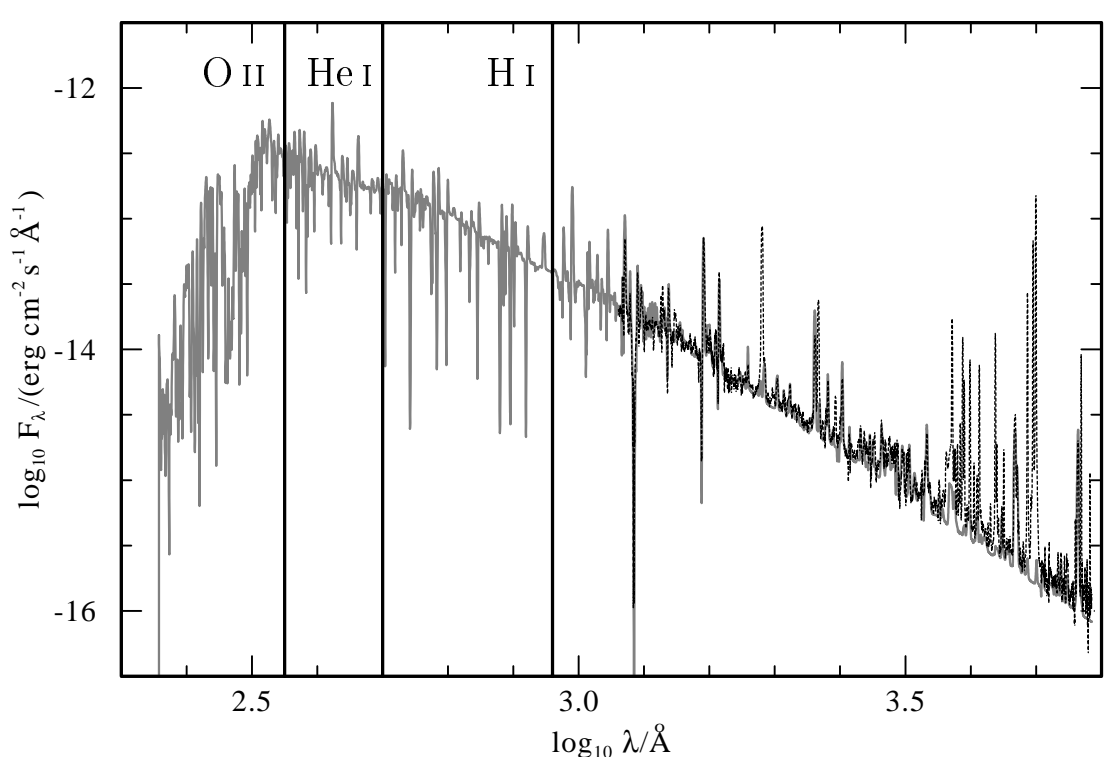

Fig. 5. Overall flux distribution and ionizing fluxes of SMP 61. The model flux is shown in grey together with the extinction-corrected observed stellar flux of SMP 61 (black, dashed). The edge frequencies for the ionization of $\mathrm{HI}, \mathrm{He}$ I and $\mathrm{O}$ II are indicated by vertical lines. as will be clear later, our initial models rather show an excess of heating.

We first determine the density distribution that reproduces the observed surface brightness distribution. By trial and error, we find that the observed surface brightness profile is well reproduced by a spherical model with a hydrogen density distribution described by

$n=n_{0} \exp \left[-\left(\frac{r-5 \times 10^{16}}{1.84 \times 10^{17}}\right)^{2}\right]$,

where $r$ is the distance to the star in $\mathrm{cm}$ and $n_{0}$ is the density at the inner boundary.

The chemical composition with which we start is the one derived by classical empirical methods in Sect. 2.2. (the abundances of $\mathrm{Mg}, \mathrm{Si}, \mathrm{Fe}$ with respect to $\mathrm{H}$ are fixed at an arbitrary value of $10^{-6}$ and do not affect our results). In a first step, we aim at reproducing the main characteristics of the nebula, which can be summarized by the following quantities: $F(\mathrm{H} \beta)$ the total nebular flux in $\mathrm{H} \beta$, the nebular outer angular radius, $\theta$, defined as the radius of the nebular image at zero intensity, and several line ratios: [S II] $\lambda 6731 / 6717$ to test the gas density, [O III] $\lambda 4363 / 5007$ to test the gas temperature in the $\mathrm{O}^{++}$zone, [O II] $\lambda 3726,3729 /[\mathrm{OII}] \lambda 7320,7330$, to test the density and temperature in the $\mathrm{O}^{+}$zone; [O III] $\lambda 5007 /\left[\mathrm{O}_{\mathrm{II}}\right] \lambda 3727$ to test the ionization structure; [O III] $\lambda 5007 / \mathrm{H} \beta$ to test the heating power of the central star, and $[\mathrm{OI}] \lambda 6300 / \mathrm{H} \beta$ to test the importance of a warm neutral zone. It is only when we are reasonably satisfied with these constraints (which we will refer to as the restricted set) that we modify the elemental abundances, if needed, to obtain an acceptable model.

In order to easily visualize whether a model is acceptable, we compute for each constraint $P$ the quantity

$\sigma_{\mathrm{P}}=\left(\log P_{\text {mod }}-\log P_{\text {obs }}\right) / t$,

where $P_{\text {mod }}$ is the value returned by the model, $P_{\mathrm{obs}}$ is the observed value, and $t$ the accepted tolerance in dex for this constraint. We determine the values of $t$ for each constraint from the estimated uncertainties in line fluxes, including the uncertainty in the dereddening process. Note that we use the outer nebular radius $\theta$ as a convenient way to visualize the constraint on the nebular size (but in fact we require the entire brightness profile to be reproduced by the model). Rather than using line fluxes with respect to $\mathrm{H} \beta$, as is done usually, we prefer to use line ratios that are easier to interpret. Apart from the traditional temperature and density diagnostics mentioned above, we also use [S II] $\lambda 4069,4076 /[$ S II] $\lambda 6717,6731$ and $\mathrm{O}$ III] $\lambda 1661 /[\mathrm{O} \mathrm{III}] \lambda 5007$, which increase with nebular temperature and density, and C III] $\lambda 1909 / C_{\text {II }} \lambda 4267$, which increases with nebular temperature. The line ratios [O III $] \lambda 5007 /[\mathrm{O}$ II $] \lambda 3727, \quad$ C III] $\lambda 1909 / \mathrm{C} \mathrm{II}] \lambda 2326$, $\mathrm{N}$ III] $\lambda 1750 /[\mathrm{N} \mathrm{II}] \lambda 6584, \quad$ [S III] $\lambda 6312 /[\mathrm{S} \mathrm{II}] \lambda 6717,6731$, and $[\mathrm{Ar}$ IV] $\lambda 4740 /[\mathrm{Ar}$ III $] \lambda 7136$ reflect the ionization structure of the nebula. Finally, [N II] $\lambda 6584 /[\mathrm{O}$ II $] \lambda 3727$, [S II] $\lambda 6725 /[\mathrm{O}$ II $] \lambda 3727, \quad$ C III] $\lambda 1909 /[\mathrm{O}$ III] $\lambda 5007$, [Ne III] $\lambda 3869 /[\mathrm{O} \mathrm{IIII}] \lambda 5007$ and [Ar III] $\lambda 7136 /[\mathrm{O}$ III $] \lambda 5007$ are closely linked to the abundances of the elements with respect to oxygen while the IRAS fluxes reflect the abundance and temperature of dust. The entire list of constraints used in this study, together with the associated tolerances is given in Table 4. Note that we do not consider redundant lines, such as [N II] $\lambda 6548$ whose ratio with [N II] $\lambda 6584$ is independent of the physical conditions in the nebula. The results of our models are presented in graphical form in the upcoming figures, where the values of $\sigma_{\mathrm{P}}$ are plotted for all the constraints $P$. The quality of a model is then easily judged by the location of the points with respect to the line of ordinate 0 . Note that we consider a model as fully satisfying only if each of the values of $\sigma_{\mathrm{P}}$ is roughly found between -1 and +1 . Any strong deviation requires an explanation.

\section{Results from the nebular model fitting}

\subsection{First models}

We use the stellar atmosphere model described above as an input to the photoionization code. From the point of view of the nebular diagnostics available, there is no difference 
Table 4. Observational constraints and tolerances for the planetary nebula SMP 61.

\begin{tabular}{|c|c|c|}
\hline$F(\mathrm{H} \beta) / 10^{-13}$ & 3.71 & 0.18 \\
\hline$\theta$ & $3.50 \times 10^{-1}$ & 0.06 \\
\hline He I $5876 / \mathrm{H} \beta$ & $1.60 \times 10^{-1}$ & 0.06 \\
\hline$[\mathrm{S}$ II $] \lambda 6731 / 6717$ & 1.81 & 0.11 \\
\hline [O III] $\lambda 4363 / 5007$ & $7.15 \times 10^{-3}$ & 0.08 \\
\hline$[\mathrm{N}$ II $] \lambda 5755 / 6584$ & $2.22 \times 10^{-2}$ & 0.30 \\
\hline [O II] $\lambda 7320,7330 /[\mathrm{O}$ II] $\lambda 3726,3729$ & $1.79 \times 10^{-1}$ & 0.08 \\
\hline [S II] $\lambda 4069,4076 /[\mathrm{S}$ II $] \lambda 6717,6731$ & $4.92 \times 10^{-1}$ & 0.18 \\
\hline O III] $\lambda 1661 /[\mathrm{O}$ III] $\lambda 5007$ & $9.18 \times 10^{-3}$ & 0.30 \\
\hline C III] $\lambda 1909 /$ C II $\lambda 4267$ & $1.76 \times 10^{2}$ & 0.15 \\
\hline [O III] $\lambda 5007 /[\mathrm{O}$ II] $\lambda 3727$ & 9.26 & 0.08 \\
\hline C III] $\lambda 1909 / C$ II] $\lambda 2326$ & 5.10 & 0.11 \\
\hline $\mathrm{N}$ III $] \lambda 1750 /[\mathrm{N} \mathrm{III}] \lambda 6584$ & $2.86 \times 10^{-1}$ & 0.30 \\
\hline [S III] $\lambda 6312 /[\mathrm{S}$ II] $\lambda 6717,6731$ & $2.03 \times 10^{-1}$ & 0.15 \\
\hline [Ar IV] $\lambda 10^{-1} 4741 /[\mathrm{Ar}$ III] $\lambda 7135$ & $4.55 \times 10^{-2}$ & 0.30 \\
\hline$[\mathrm{O}$ III $] \lambda 5007 / \mathrm{H} \beta$ & 7.41 & 0.04 \\
\hline$[\mathrm{O} \mathrm{I}] \lambda 6300 / \mathrm{H} \beta$ & $4.20 \times 10^{-2}$ & 0.15 \\
\hline$[\mathrm{N}$ II $] \lambda 6584 /[\mathrm{O}$ II $] \lambda 3727$ & $3.94 \times 10^{-1}$ & 0.08 \\
\hline [S II] $\lambda 6725 /[\mathrm{O}$ II] $\lambda 3727$ & $7.37 \times 10^{-2}$ & 0.15 \\
\hline C III] $\lambda 1909 /[$ O III] $\lambda 5007$ & $4.26 \times 10^{-1}$ & 0.11 \\
\hline$[\mathrm{Ne}$ III $] \lambda 3869 /[\mathrm{O}$ III $] \lambda 5007$ & $5.67 \times 10^{-2}$ & 0.06 \\
\hline$[\mathrm{Ar}$ III $] \lambda 7135 /[\mathrm{O}$ III] $] \lambda 5007$ & $1.19 \times 10^{-2}$ & 0.10 \\
\hline$F(12 \mu \mathrm{m})$ & $8.00 \times 10^{-2}$ & 0.18 \\
\hline$F(25 \mu \mathrm{m})$ & $1.30 \times 10^{-1}$ & 0.10 \\
\hline$F(60 \mu \mathrm{m})$ & $1.60 \times 10^{-1}$ & 0.18 \\
\hline
\end{tabular}

between using the model atmosphere with iron mass fraction $X_{\mathrm{Fe}}=4 \times 10^{-4}$ or $X_{\mathrm{Fe}}=5 \times 10^{-5}$. The hydrogen density distribution of the nebular model is given by Eq. (1). As will be made clear later on, one of the main shortcomings of the models is that the excitation, as measured by [O III] $\lambda 5007 /[\mathrm{O}$ II] $\lambda 3727$ is too high. We therefore discuss only models tailored to provide the lowest possible [O III] $\lambda 5007 /[\mathrm{O}$ II] $\lambda 3727$ ratio. For example, there is no point to present models in which the nebula would have an inner radius smaller than $5 \times 10^{16} \mathrm{~cm}$ (which corresponds to $0.065^{\prime \prime}$ at the distance of the LMC). Also, while planetary nebulae are not necessarily ionization bounded, optically thin models are not discussed, since they lead to higher [O III] $\lambda 5007 /[\mathrm{O}$ II $] \lambda 3727$ ratios than ionization-bounded models. We tried various values of the inner density $n_{0}$. Note that the [S II] $\lambda 6731 / 6717$ ratio is not very sensitive to $n_{0}$, due to the fact that the hydrogen density decreases outwards, and that, in addition, the electron density in the [S II] 66725 emitting zone is smaller than the hydrogen density. We show in Fig. 6 a series of models with $n_{0}$ equal to $1.0 \times 10^{4} \mathrm{~cm}^{-3}$ (circles), $1.05 \times 10^{4} \mathrm{~cm}^{-3}$ (squares), $1.1 \times 10^{4} \mathrm{~cm}^{-3}$ (triangles), $1.15 \times 10^{4} \mathrm{~cm}^{-3}$ (diamonds). All these models have a dust-to-gas mass ratio of $3 \times 10^{-4}$. We see that a value of $n_{0}$ between about 1.02 and $1.12 \times 10^{4} \mathrm{~cm}^{-3}$ leads to an angular radius (and a surface brightness profile) compatible with the observations. But they give too large $F(\mathrm{H} \beta)$ (by a factor about 3 compared to the observed value after correction for extinction). We cannot help by adding more grains to absorb part of the Lyman continuum radiation, since the modelled flux in the $25 \mu \mathrm{m}$ IRAS band is already

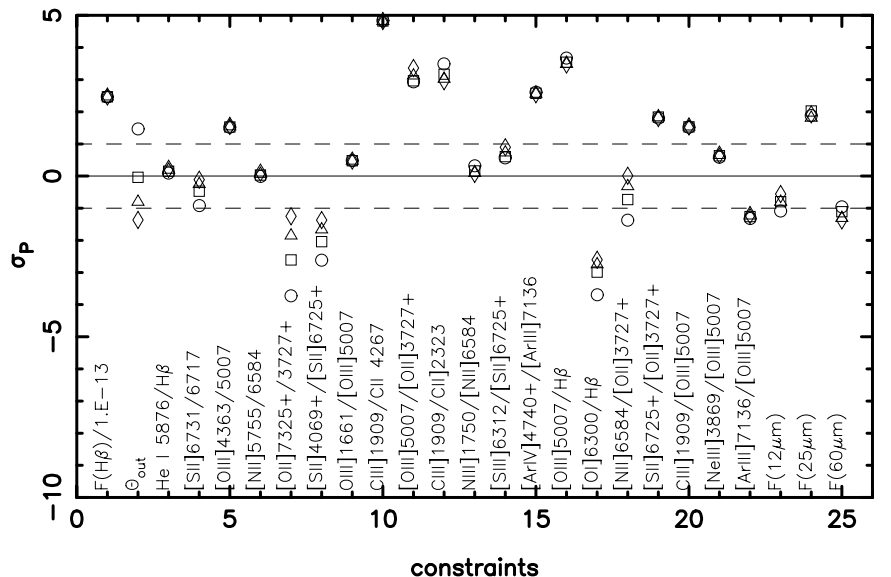

Fig. 6. Representation of a series of photoionization models of SMP 61. The plotted quantities are the values of $\sigma_{\mathrm{P}}=$ $\left(\log P_{\text {mod }}-\log P_{\text {obs }}\right) / t$ as a function of the observational constraint $P$ (see text in Sect. 5.2). The photoionization models have a density structure that reproduces the observed surface brightness profile and they use as an input the stellar atmosphere flux described in Sect. 4. The chemical composition of these models is described in Sect. 2.2. All the nebular models in this figure have a volume filling factor of one. Models with inner density $n_{0}=1.0 \times 10^{4}, 1.05 \times 10^{4}, 1.1 \times 10^{4}$, $1.15 \times 10^{4} \mathrm{~cm}^{-3}$ are represented by circles, squares, triangles and diamonds respectively. In principle, a completely satisfactory model should have all its points located between the dashed lines.

larger than observed. Thus, we conclude that, either the real number of stellar photons in the Lyman continuum is smaller than in the model atmosphere or that there is some leakage of ionizing photons. As seen in Fig. 6, the photoionization models also predict too large [O III] $\lambda 5007 /[\mathrm{O} \mathrm{II}] \lambda 3727$ and [O III $] \lambda 5007 / \mathrm{H} \beta$ (with a $\sigma_{\mathrm{P}}$ of $3-4$ ), and slightly too large [O III] $\lambda 4363 / 5007$. This cannot be improved by changing the oxygen abundance or by fine tuning the $\mathrm{C} / \mathrm{O}$ and $\mathrm{S} / \mathrm{O}$ ratios to fit the observed C III] $\lambda 1909 /[\mathrm{O}$ III] $\lambda 5007$ and $[\mathrm{S} \mathrm{II}] \lambda 6725 /[\mathrm{O}$ II $] \lambda 3727$ ratios. The models also have too low [O II] $\lambda 7320,7330 /[\mathrm{O}$ II $] \lambda 3726,3729$ and [S II] $\lambda 4069,4076 /[\mathrm{S} \mathrm{II}] \lambda 6717,6731$ ratios, the agreement with the observations being better for higher values of $n_{0}$, because the nebula is then more efficient in absorbing photons and the ionization front occurs at a higher value of the density.

In order to get away with the $F(\mathrm{H} \beta)$ problem, one could argue that the covering factor of the nebula is smaller than unity, so that some ionizing photons escape without interacting with the gas. But this would not help in solving the $[\mathrm{O}$ III $] \lambda 5007 /[\mathrm{O} \mathrm{II}] \lambda 3727,[\mathrm{O}$ III $] \lambda 5007 / \mathrm{H} \beta$ and [O III] $\lambda 4363 / 5007$ problems.

We do not attempt two-sector models of different densities (such as those of Clegg et al. 1987 or Dudziak et al. 2000) for the following reasons. First, as mentioned in Sect. 2, the nebular image is extremely round. This, a priori does not mean that the nebula is spherical. Indeed, it could be elongated but seen pole on. But, in that case, the nebular extension along the line of sight would be larger than the one observed on the images. Replacing a portion of the model nebula with two sectors of lower density (to obtain a larger dimension) would considerably worsen the [O II] $\lambda 7320,7330 /[\mathrm{O}$ II] $\lambda 3726,3729$ 


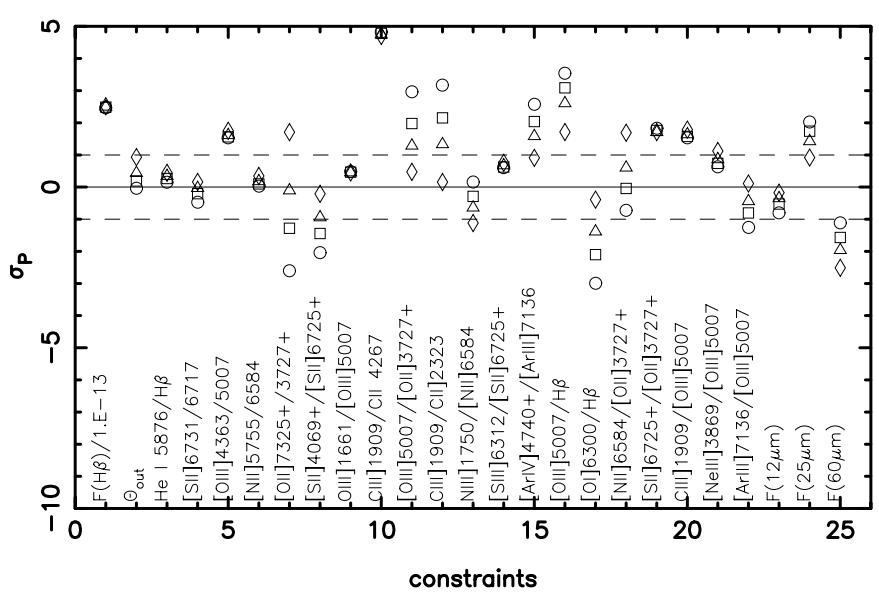

Fig. 7. Same as Fig. 6 but for models with different filling factors. Models defined by $\left(n_{0}, \epsilon\right)$ equal to $\left(1.05 \times 10^{4} \mathrm{~cm}^{-3}, 1.0\right)$ are represented by circles, $\left(1.5 \times 10^{4} \mathrm{~cm}^{-3}, 0.49\right)$ by squares, $\left(2.0 \times 10^{4} \mathrm{~cm}^{-3}\right.$, 0.27 by triangles, $\left(3.0 \times 10^{4} \mathrm{~cm}^{-3}, 0.122\right)$ by diamonds.

and [S II] $\lambda 4069,4076 /[\mathrm{S} \mathrm{II}] \lambda 6717,6731$ ratios while not helping much for the $[\mathrm{O}$ III $] \lambda 5007 /[\mathrm{O}$ II $] \lambda 3727$ ratio and worsening the $[\mathrm{O} \mathrm{III}] \lambda 5007 / \mathrm{H} \beta$ ratio, as we can judge from spherical models built at lower densities. Besides, if the low density sector is density bounded, this may indeed help with the $\mathrm{H} \beta$ flux, but will worsen the $[\mathrm{O}$ III $] \lambda 5007 /[\mathrm{O} \mathrm{II}] \lambda 3727$ ratio.

One way to lower the excitation of the nebula is to assume that it is clumpy. Models that differ in density and filling factor but have the same value of the product $n_{0}^{2} \epsilon$, where $\epsilon$ is the volume filling factor, will have about the same external radius but will have lower excitation for higher densities. Such a series of models is shown in Fig. 7, where the values of the couple $\left(n_{0}, \epsilon\right)$ are $(1.05 \times$ $\left.10^{4} \mathrm{~cm}^{-3}, 1.0\right)$ (circles), $\left(1.5 \times 10^{4} \mathrm{~cm}^{-3}, 0.49\right)$ (squares), $\left(2.0 \times 10^{4} \mathrm{~cm}^{-3}, 0.27\right)$ (triangles), $\left(3.0 \times 10^{4} \mathrm{~cm}^{-3}, 0.122\right)$ (diamonds). As seen in the figure, models with higher densities considerably improve many of the line ratios, and especially the $[\mathrm{O}$ III] $\lambda 5007 /[\mathrm{O}$ II $] \lambda 3727$ and $[\mathrm{O}$ III $] \lambda 5007 / \mathrm{H} \beta$ ratios as well as the [O II] $\lambda 7320,7330 /[\mathrm{OII}] \lambda 3726,3729$ and [S II] $\lambda 4069,4076 /[\mathrm{S} \mathrm{II}] \lambda 6717,6731$ ratios and also the [O I] $\lambda 6300 / \mathrm{H} \beta$ ratio. It is then easy to fine tune the $\mathrm{N}, \mathrm{S}$ and $\mathrm{C}$ abundances. A model with $n_{0}$ between $2.0 \times 10^{4}$ and $3.0 \times$ $10^{4} \mathrm{~cm}^{-3}$ would then be in agreement with all the observational constraints (except C III] $\lambda 1909 / C_{\text {II }} \lambda 4267$, but see later) if one assumed a covering factor of roughly $1 / 3$.

The predicted emission in the IRAS bands deserves some comments. First, we note in Fig. 7 that models with lower excitation show lower fluxes in the IRAS bands. The reason is that the larger proportion of neutral hydrogen particles in the nebula reduces the amount of Lyman continuum photons absorbed by dust. Another point to make is that, in the hypothesis of incomplete coverage of the ionizing source by the nebula (and if dust is intimately mixed with the gas), the predicted IRAS fluxes should be larger than observed by roughly the same amount as $F(\mathrm{H} \beta)$ is larger than observed in our models computed for a covering factor of unity. Obviously, this is not the case of our predicted flux in the $12 \mu \mathrm{m}$ band (the observed flux in the $60 \mu \mathrm{m}$ band is only an upper limit so there is no problem there). Increasing the dust abundance only worsens the problem, since then the dust particles are more numerous to share the photons that heat them, and the temperature of the dust grains becomes smaller, decreasing the flux in the $12 \mu \mathrm{m}$ band with respect to that in the $25 \mu \mathrm{m}$ band. Actually, there may be a simple explanation to our under-prediction of the flux in the $12 \mu \mathrm{m}$ band. Our code does not handle PAHs, while PAHs are known to exist in planetary nebulae with Wolf-Rayet central stars (Szczerba et al. 2001; Cohen 2001) and they contribute to the emission in the $12 \mu \mathrm{m}$ band. Note also that our predictions concerning dust emission are given here only for the ionized part of the nebula. The presence of dust in the neutral zone would increase the fluxes in the $25 \mu \mathrm{m}$ and $60 \mu \mathrm{m}$ bands. We do not consider this in the modelling presented here, since it brings no useful information to the problem we are interested in. In the following, we therefore simply require from our models that they do not over-predict the $12 \mu \mathrm{m}$ and $25 \mu \mathrm{m}$ fluxes with respect to the observations.

We have found that with a model having an inner density of about 2-3 $\times 10^{4} \mathrm{~cm}^{-3}$ and a filling factor about $0.3-0.1$, we are able to reproduce many of the observational constraints, provided that we assume a covering factor of about $1 / 3$. However, this model is still not entirely satisfactory. Mainly, the [O III] temperature is still too high and so is the predicted [O III] $\lambda 5007 / \mathrm{H} \beta$ ratio. And a conspicuous shortcoming of the model is that the C II $\lambda 4267 / \mathrm{C} \mathrm{III} \lambda 1909$ ratio is predicted too large by a factor of about 5 . Note that, while the density structure of planetary nebulae is known generally not to be uniform on a small scale from high resolution images, the clumps or filaments of gas are actually embedded in a more diffuse medium. This diffuse medium is expected to be more highly ionized, and thus its presence will enhance the [O III] $\lambda 5007 /[\mathrm{O}$ II $] \lambda 3727$ predicted by our simple model. We do not attempt to construct a two-phase model but we keep this in mind.

In the following, we look for more satisfactory solutions by releasing some of the assumptions of our models.

\subsection{Models with modified stellar fluxes}

Due to the compact and spherical appearance of the nebula, the covering factor of $1 / 3$ which is needed to explain $F(\mathrm{H} \beta)$ seems to be very unrealistic. The obvious next step is therefore to assume that the discrepancy is at least partly due to an overestimation of the stellar ionizing radiation field in Sect. 4. As explained in Sect. 4.2, the error for the derived stellar luminosity may be as high as a factor of 2 under very pessimistic assumptions.

Let us therefore first consider the case where we assume that the number of photons emitted by the star at any energy larger than $13.6 \mathrm{eV}$ is only $50 \%$ of what is predicted by the model atmosphere (from the point of view of the photoionization model, it is equivalent to assume that the star luminosity is lower than given in Sect. 4 by a factor 2). The results are shown in Fig. 8. Here, the values of the couple $n_{0}, \epsilon$ are $7.5 \times 10^{3} \mathrm{~cm}^{-3}$, 1.0 (circles), $1.0 \times 10^{4} \mathrm{~cm}^{-3}, 0.6$ (squares), $1.2 \times 10^{4} \mathrm{~cm}^{-3}$, 0.4 (triangles), $1.4 \times 10^{4} \mathrm{~cm}^{-3}, 0.3$ (diamonds) while the dustto-gas mass ratio is still assumed to be $3 \times 10^{-4}$. Since the 


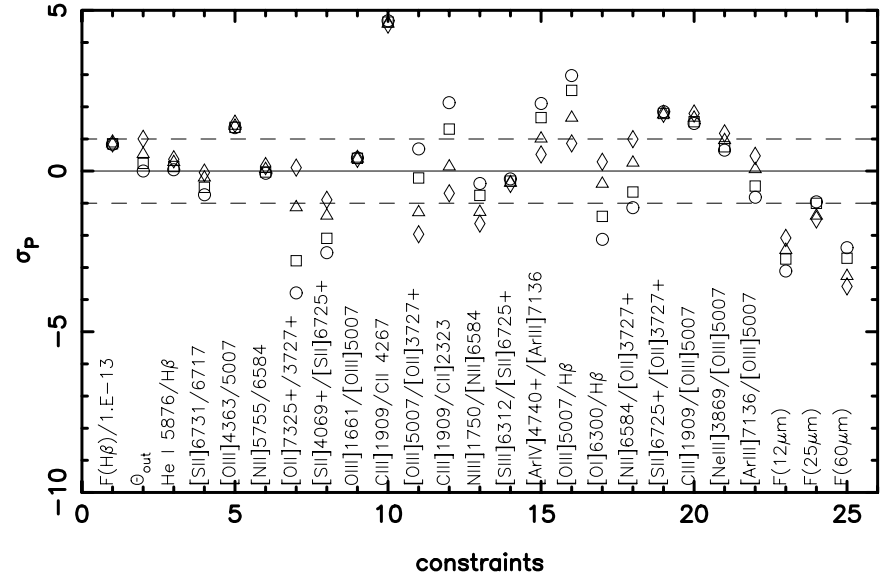

Fig. 8. Same as Fig. 7 but for models with in which the stellar photon flux has been divided by a factor 2 with respect to the original model for all the energies larger than $13.6 \mathrm{eV}$. Models defined by $\left(n_{0}, \epsilon\right)$ equal to $\left(7.5 \times 10^{3} \mathrm{~cm}^{-3}, 1.0\right)$ are represented by circles, $\left(1 \times 10^{4} \mathrm{~cm}^{-3}, 0.6\right)$ by squares, $\left(1.2 \times 10^{4} \mathrm{~cm}^{-3}, 0.4\right)$ by triangles, $\left(1.4 \times 10^{4} \mathrm{~cm}^{-3}, 0.3\right)$ by diamonds.

number of ionizing photons is smaller than in the previous case, we had to take smaller densities to match the observed size of the ionized nebula. Globally, the ionization parameter is nevertheless smaller, so that [O III] $\lambda 5007 /[\mathrm{O} \mathrm{II}] \lambda 3727$ can now be fitted even with a model with a filling factor of unity. Of course, the [O II] $\lambda 7320,7330 /[\mathrm{OII}] \lambda 3726,3729$ and [S II] $\lambda 4069,4076 /[\mathrm{S} \mathrm{II}] \lambda 6717,6731$ ratios are better reproduced for the highest density models of this series, i.e. $n_{0}$ around $1.5 \times 10^{4} \mathrm{~cm}^{-3}$, which again require a small filling factor. The best models of this series are at least as good as the best models shown in Fig. 7 without requiring a small covering factor. Note that the [O III] $\lambda 4363 / 5007$ ratio is still somewhat high in this series of models, and adjusting the $\mathrm{S}$, $\mathrm{C}$ and $\mathrm{Ne}$ abundances (by lowering them) can only make the problem worse.

Another option to consider is a radiation field softened in the Lyman continuum. This would not only lower the [O III $] \lambda 5007 /[\mathrm{O}$ II $] \lambda 3727$ ratio, but also reduce the heating. Figure 9 shows a series of models identical to those of Fig. 8, but this time keeping $60 \%$ of the photons between 13.6 and $24.6 \mathrm{eV}$ and $40 \%$ of the photons with energies larger than $24.6 \mathrm{eV}$. Clearly, the models from this series represented by the triangles are quite satisfactory also as regards the electron temperature and the $[\mathrm{O}$ III $] \lambda 5007 / \mathrm{H} \beta$ ratio. [S II] $\lambda 6725 /[\mathrm{O}$ II] $\lambda 3727$ can easily be matched by reducing the original sulfur abundance by a factor 1.3-2). The observed fluxes in the IRAS $25 \mu \mathrm{m}$ and $60 \mu \mathrm{m}$ bands are compatible with dust-to-gas mass ratios of up to $3 \times 10^{-3}$. The quality of the fit of the optical and UV data is not affected by the amount of dust in the allowed range. The only important mismatch of the model with the observations is the C III] $\lambda 1909 / C_{\text {II }} \lambda 4267$ whose computed value is much higher than observed, by a factor of almost 4. This problem is discussed in the following subsection.

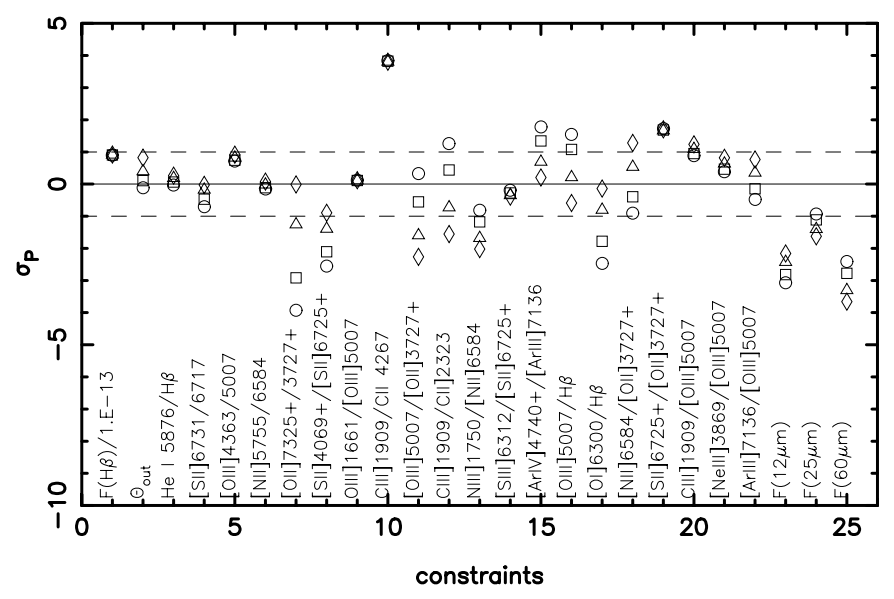

Fig. 9. The same as Fig. 8 for models ionized by a softened stellar flux (see text). Models defined by $\left(n_{0}, \epsilon\right)$ equal to $\left(7.5 \times 10^{3} \mathrm{~cm}^{-3}, 1.0\right)$ are represented by circles, $\left(1 \times 10^{4} \mathrm{~cm}^{-3}, 0.6\right)$ by squares, $\left(1.2 \times 10^{4} \mathrm{~cm}^{-3}\right.$, $0.4)$ by triangles, $\left(1.4 \times 10^{4} \mathrm{~cm}^{-3}, 0.3\right)$ by diamonds.

\subsection{Models with inhomogeneous nebular composition}

It is a known fact that recombination lines of $\mathrm{C}$, and also of other elements like $\mathrm{N}, \mathrm{O}, \mathrm{Ne}$ generally indicate much higher abundances of the parent ions than collisionally excited lines (see e.g. Liu 2002 or Esteban 2002 and references therein). Many explanations have been proposed (see e.g. Stasińska 2002 for a review). The generally preferred one is to assume that the chemical composition of the nebulae is not uniform (e.g. Torres-Peimbert et al. 1990; Liu et al. 2000). And indeed, there is direct evidence at least in two nebulae (A 30 and A 78) of the presence of clumps of matter enriched in C (Jacoby \& Ford 1983; Harrington \& Feibelman 1984) and also N, O and Ne for A30 (Wesson et al. 2003). The central star of SMP 61 being a Wolf-Rayet, this nebula is a good candidate for carbon enrichment. If material seen in the stellar wind ends up in the nebula as cool clumps, these clumps will strongly emit in the C II $\lambda 4267$ line. We have mimicked such a situation by assuming that the inner zone of the nebula consists of helium- and carbon-rich material, maintaining the same density distribution as before. The number of combinations to explore is obviously very high, and we have run many simulations. In Fig. 10, we present the result of a series of simulations with $n_{0}=1.4 \times 10^{4} \mathrm{~cm}^{-3}, \epsilon=0.3$ (the best combination from Fig. 9) with the following abundances of He and $\mathrm{C}$ (relative to $\mathrm{H}$ by number) in the inner $3 \times 10^{-3} M_{\odot}$ of the nebula: 1.04 and 0.4 (circles), 0.78 and 0.3 (squares), 0.52 and 0.2 (triangles), 0.26 and 0.1 (diamonds). These ratios correspond to the mass ratio of $\mathrm{He}$ and $\mathrm{C}$ in the atmosphere of the central star, as derived in Sect. 4. Otherwise, the number abundance ratios with respect to hydrogen are: $\mathrm{H}: \mathrm{He}: \mathrm{C}: \mathrm{N}: \mathrm{O}: \mathrm{Ne}$ : $\mathrm{S}: \mathrm{Ar}=1: .09: 4.0 \times 10^{-4}: 3.95 \times 10^{-5}: 2.65 \times 10^{-4}: 4.26 \times 10^{-5}$ : $4.0 \times 10^{-6}: 8 \times 10^{-7}$. We see that the last combination provides a very good fit to the data, including the C II] $\lambda 1909 /$ C II $\lambda 4267$ line ratio. Probably, other combinations can be found that provide a similar fit. 


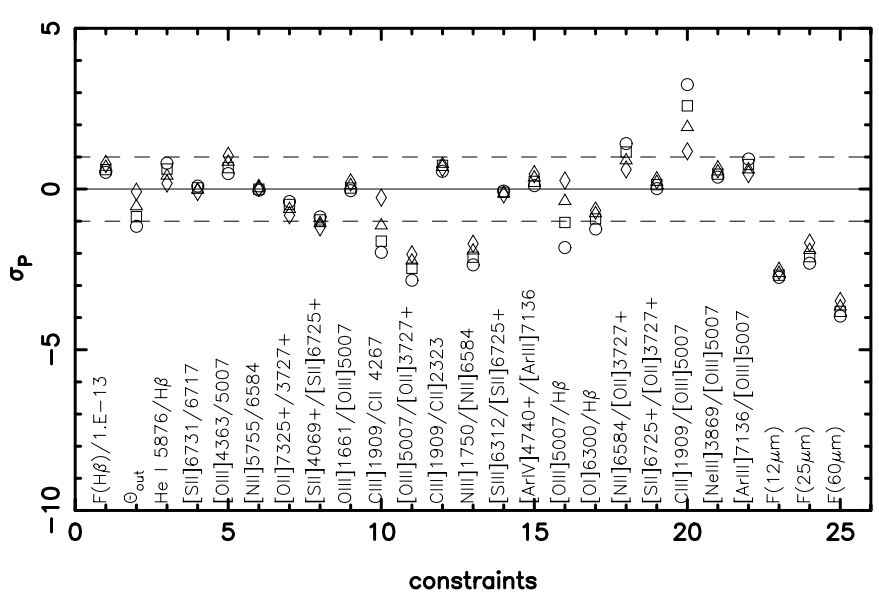

Fig. 10. Models ionized by a softened stellar flux (as in Fig. 9), with an inner density $n_{0}=1.4 \times 10^{4} \mathrm{~cm}^{-3}$ and a nebular filling factor $\epsilon=$ 0.3 (the best combination of Fig. 9). Now, the inner $3 \times 10^{-3} M_{\odot}$ of the nebula are He- and C-rich. The abundances of $\mathrm{He}$ and $\mathrm{C}$ (by number relative to $\mathrm{H}$ ) are: 1.04 and 0.4 (circles), 0.78 and 0.3 (squares), 0.52 and 0.2 (triangles), 0.26 and 0.1 (diamonds). Otherwise, the abundance ratios with respect to hydrogen are (by number): $\mathrm{H}: \mathrm{He}: \mathrm{C}: \mathrm{N}$ : O: Ne: S: $\mathrm{Ar}=1: .09: 4.0 \times 10^{-4}: 3.95 \times 10^{-5}: 2.65 \times 10^{-4}: 4.26 \times 10^{-5}$ : $4.0 \times 10^{-6}: 8 \times 10^{-7}$.

\section{Discussion}

\subsection{Stellar ionizing fluxes}

The nebular analysis of SMP 61 indicates two possible problems concerning the theoretical flux distribution of the central star: The absolute number of Lyman photons is probably too large by a factor of 2, and the Lyman continuum radiation field is possibly too hard. The first problem may be discussed away by assuming a very pessimistic value for the uncertainty of the derived luminosity. However, the second point concerns the slope of the energy distribution in the flux maximum and cannot be easily resolved.

A simultaneous solution of both problems would be achieved by decreasing the effective temperature $T_{\text {eff }}$ of the model atmosphere (i.e. increasing the radius where $\tau_{\text {Ross }}=$ $2 / 3$ ). In this way, photons would be distributed from the flux maximum to the observed wavelength range. In effect, the derived luminosity would be decreased, and a softer flux distribution would be obtained in the flux maximum. For a corresponding decrease of $T_{\text {eff }}$, the stellar core temperature must be lowered significantly, or the mass-loss rate must be increased. Of course, both operations are strongly limited by the necessity to fit the observed spectrum of the central star. Test calculations show that the simultaneous fitting of the observed spectrum and the nebular ionizing fluxes is not possible with the model atmospheres applied in present work.

A possible solution concerns the treatment of dielectronic recombination in our stellar atmosphere code. In the present version dielectronic transitions are treated as optically thin, and their contribution to the rate equations is accounted for by the approach of Mihalas (1971). However, when C IV recombines to $\mathrm{C} \mathrm{III} \mathrm{in} \mathrm{the} \mathrm{outer} \mathrm{part} \mathrm{of} \mathrm{the} \mathrm{WC} \mathrm{atmosphere,} \mathrm{the} \mathrm{ioniza-}$ tion edge of $\mathrm{C}$ III becomes optically thick. In this case also the corresponding dielectronic transitions become optically thick, and their recombination efficiency is reduced because recombination photons cannot escape. Indeed, first tests with an improved treatment show that the recombination from C IV to C III is suppressed by this effect, leading to higher derived mass-loss rates and slightly lower stellar temperatures. A detailed investigation of this topic will be presented in a forthcoming paper.

\subsection{The effect of He-rich clumps on the nebular spectrum}

He-rich clumps, which we have invoked in Sect. 6.3, can in fact soften the radiation field available to the rest of the nebula under certain conditions, by selectively absorbing the photons above $24.6 \mathrm{eV}$. The total mass of helium $M_{\mathrm{He}}^{\mathrm{a}}$ required to absorb all the $\mathrm{He}^{0}$-ionizing photons emitted by the central star is of the order $10^{3} / n_{\mathrm{e}} M_{\odot}$ (where $n_{\mathrm{e}}$ is the electron density in $\mathrm{cm}^{-3}$ ). If the integrated mass of helium in the clumps is at least equal to this value and if the clumps are located close to the inner boundary of the nebula with an integrated covering factor of unity, then the $\mathrm{He}^{0}$-ionizing photons would be completely blocked by the He-rich material. Such an extreme situation does not correspond to the case of SMP 61, since [O III] $\lambda 5007$ is emitted not only in the most central part but in the entire nebula. One can however imagine a less extreme situation where the high density He- and C-rich clumps are distributed over the entire volume of the nebula and soften the average radiation field available to the bulk of the nebular material. In such a case, a softening of the predicted stellar energy distribution in the Lyman continuum would perhaps not be necessary. Note however that such clumps are not efficient in absorbing radiation between 13.6 and $24.6 \mathrm{eV}$, unless they are very dusty, which for SMP 61 seems excluded by the observed IRAS fluxes. Obviously, the impact of these He- and C-rich clumps on the global spectrum of the nebula will strongly depend on their density, on the amount of mixing with nebular material and on their spatial distribution in the nebula.

In the case of SMP 61, we can estimate the maximum mass of helium contained in the clumps by considering that the $\mathrm{He} / \mathrm{C}$ proportion is the same as in the stellar wind and by using the observed flux in the C II $\lambda 4267$ line. The observed flux in this line corresponds to a carbon mass $M_{\mathrm{C}}$ of about $10 / n_{\mathrm{e}} M_{\odot}$, if most of the carbon is in $\mathrm{C}^{++}$form and if the line is emitted at a temperature of $8000 \mathrm{~K}$, which is the case for the C-rich zone in our models. Since helium and carbon are roughly equal by mass in the stellar wind, this means that the total helium mass is much smaller than to $M_{\mathrm{He}}^{\mathrm{a}}$, implying that there is no significant blocking of $\mathrm{He}^{0}$-ionizing photons by the clumps. If the clumps are of much higher density than in our models, $\mathrm{C}^{++}$may be partly recombined in the clumps, increasing $M_{\mathrm{C}}$ with respect to $M_{\mathrm{He}}^{\mathrm{a}}$, but in that case the clumps will have a smaller cross section and will be less efficient to block the stellar radiation. Therefore, in the specific case of SMP 61, we do not think that the He-rich material will significantly soften the stellar radiation available to the nebula. 


\subsection{Stellar parameters and evolutionary status}

The derived stellar parameters are in line with recent analyses of galactic [WC]-type central stars based on line-blanketed models (De Marco \& Crowther 1998, 1999; De Marco et al. 2001; Crowther et al. 2003). In contrast to previous unblanketed calculations (Koesterke \& Hamann 1997a,b), the new models show a trend towards similar surface mass fractions for early and late [WC] subtypes, with $X_{\mathrm{O}} \approx 10 \%$ and $X_{\mathrm{C}} / X_{\mathrm{He}} \approx 1$.

Due to the known distance to the LMC, SMP 61 offers the rare chance to determine the luminosity of a [WC]-type central star. From the analysis of the stellar spectrum alone we infer a value of $10^{3.9} L_{\odot}$. The nebular analysis reveals that this value is probably too large by up to $0.3 \mathrm{dex}$ - dependent on the assumed nebular covering factor. The luminosity of SMP 61 is therefore in the range of $10^{3.6}-10^{3.9} L_{\odot}$. For a second object, $\mathrm{BD}+303696$, Li et al. (2002) derive a distance of $1.2 \mathrm{kpc}$ from the angular expansion of the nebula. For this distance, Crowther et al. (2003) obtain a value of $L_{\star}=10^{3.6} L_{\odot}$. Both values are in the range that is expected for the majority of all post-AGB stars, with a typical mass around $0.6 M_{\odot}$ (see Blöcker 1995; Herwig 2001b). Altogether, no evidence is found that [WC] central stars have different masses than H-rich objects although, admittedly, the number of cases with known $L_{\star}$ is small.

An interesting hint of the evolutionary status of SMP 61 is given by its low iron and nitrogen abundances. For a standard solar composition as given by Gray (1992, p. 318) or Grevesse \& Sauval (1998) a ratio of $\log (\mathrm{Fe} / \mathrm{O})=-1.33$ is expected. More recent investigations (Allende Prieto et al. 2001a; Allende Prieto et al. 2001b) imply a lower solar oxygen abundance and give $\log (\mathrm{Fe} / \mathrm{O})=-1.19$. The upper limit of $X_{\mathrm{Fe}}<1 \times 10^{-4}$ that is derived in Sect. 4 translates to $\log (\mathrm{Fe} / \mathrm{H})<-5.54$ for a solar composition. In relation to the oxygen abundance of the nebula $(\log (\mathrm{O} / \mathrm{H})=-3.58)$ we obtain $\log (\mathrm{Fe} / \mathrm{O})<-1.96$, i.e. iron is under-abundant by at least $0.63 \mathrm{dex}$ in the atmosphere of the central star. In addition, no nitrogen is detected in our analysis. The upper limit of $X_{\mathrm{N}}<5 \times 10^{-5}$ lies 0.9 dex below the abundance derived for the nebula.

The detection of an iron deficiency is in line with recent results from analyses of PG 1159 stars, the probable descendants of [WC]-type central stars. Miksa et al. (2002) set upper limits from 0 to -1.5 dex solar for the iron abundance of 15 PG 1159 stars. Additionally, Werner et al. (2003) find an iron depletion of at least -1.5 dex for the central star of A78, a [WC]-PG 1159 transition object. Also Crowther et al. (2003) find evidence for an iron abundance of $0.3-0.7$ dex below solar for BD+303696 and NGC 40. However, for these two objects the nebulae show oxygen abundances significantly below solar (see Peña et al. 2001), so that the iron deficiency may be attributed to a low initial metallicity.

Such an iron deficiency is expected for material which has been exposed to s-process nucleosynthesis in the He-intershell of thermally pulsating AGB or post-AGB stars (see Lugaro et al. 2003; Herwig et al. 2003). This region is expected to consist of partially He-burned material, i.e. mainly $\mathrm{He}, \mathrm{C}$, $\mathrm{O}$ and no N. The observed surface composition of SMP 61 therefore exactly resembles the abundance pattern that is expected for a He-intershell where the s-process has been active.

For the formation of central stars with [WC] surface composition, the H-rich layers above the He-intershell must be removed or mixed with intershell material. Herwig (2001a) and Blöcker (2001) demonstrated that the latter is possible by dredge-up of intershell material after an AGB-final thermal pulse (AFTP) or a late- or very late thermal pulse on the postAGB (LTP, VLTP). In case of a LTP or VLTP very long evolutionary timescales on the post-AGB are expected, because the central star first evolves to the blue as H-burner, then back to the red during the thermal pulse, and again to the blue as He-burning [WC] star.

The observed absence of nitrogen for SMP 61 strongly favors the AFTP scenario because for a LTP or VLTP nitrogen is produced by the $\mathrm{CNO}$-cycle in the H-burning phase. In the AFTP scenario the last thermal pulse occurs when the star is leaving the AGB, and the central star directly enters the postAGB as a He-burner. However, to obtain a sufficiently high probability for the AFTP to occur at small enough $\mathrm{H}$-envelope masses, a coupling of mass-loss to the thermal pulse cycle is required (Blöcker 2001). From the upper limit of $X_{\mathrm{N}}<5 \times 10^{-5}$ we can derive an upper limit for the mass of the remaining $\mathrm{H}$-envelope after the final thermal pulse.

Under the assumption that nitrogen has nebular abundance in the H-envelope, and is completely destroyed in the He-intershell, it follows that the maximum mass of the H-envelope can be only 0.15 of the dredged-up mass from the intershell. Herwig (2001a) finds that the dredged-up mass is typically of the order of $6 \times 10^{-3} M_{\odot}$. Consequently, the remaining $\mathrm{H}$-envelope mass at the final thermal pulse must have been smaller than $9 \times 10^{-4} M_{\odot}$. This value is significantly smaller than the corresponding envelope masses in the AFTP models of Herwig (2001a). For these models the mass-loss on the AGB has been tuned in such a way that envelope masses of $3 \times 10^{-2}$ and $4 \times 10^{-3} M_{\odot}$ were obtained after the thermal pulse. The present work implies even lower values, and therefore also lower $\mathrm{H}$-abundances for the resulting [WC]-type central stars.

Additional evidence against the LTP and VLTP scenarios is due to the very young appearance of the planetary nebula. Using the observed nebular expansion velocity of $29.3 \mathrm{~km} \mathrm{~s}^{-1}$ (Vassiliadis et al. 1998b) and angular radius from Table 4 we find an expansion time of about $3000 \mathrm{yr}$, whereas evolutionary tracks of Blöcker $(1995,2001)$ for a $0.625 M_{\odot}$ central star undergoing a VLTP imply a timescale of at least $8000 \mathrm{yr}$ to reach a temperature of $85 \mathrm{kK}$ (dependent on the time where the zero point for the PN age is assumed). Moreover, Górny \& Tylenda (2000) find general evidence against the LTP and VLTP scenarios due to the similarity of the [WR]PN and non-[WR]PN populations.

\subsection{Stellar mass-loss history}

We know from the analysis of the central star atmosphere that the presently observed mass-loss rate is $\dot{M}=10^{-6.12} M_{\odot} \mathrm{yr}^{-1}$ and that the carbon mass fraction is 0.52 (see Table 3 ). 
As mentioned in Sect. 6.3, the total mass of carbon in the nebula required to produce the observed flux in the $\mathrm{C}$ II $\lambda 4267$ line, $M_{\mathrm{C}}$, is about $10 / n_{\mathrm{e}} M_{\odot}$. In our model, the value of $n_{\mathrm{e}}$ in the zone where this line is mostly emitted is about $2 \times 10^{4} \mathrm{~cm}^{-3}$ which implies $M_{\mathrm{C}} \approx 5 \times 10^{-4} M_{\odot}$. Note that $M_{\mathrm{C}}$ is just slightly larger than the total mass of carbon in the $\mathrm{C}$-rich zone, and that, if the density of the carbon-rich clumps is larger, the total carbon mass will be smaller. We thus infer that the star has spent about $1300 \mathrm{yr}$ in a similar state of mass-loss.

This timescale is significantly lower than the time expected for a PN central star to reach $T_{\text {eff }}=85 \mathrm{kK}$. Evolutionary tracks for He-burning stars of the corresponding luminosity from Vassiliadis \& Wood (1994) give values around $3000 \mathrm{yr}$. Vassiliadis et al. (1998b) show that this timescale fits the nebular age of SMP 61. H-burning tracks from Blöcker (1995) imply an age of $3000 \mathrm{yr}$ for a post-AGB mass of $0.625 M_{\odot}$ (with $\left.L_{\star}=10^{3.9} L_{\odot}\right)$ and $5000 \mathrm{yr}$ for $0.605 M_{\odot}$ (with $\left.L_{\star}=10^{3.7} L_{\odot}\right)$.

Our modelling then suggests that the mass-loss of SMP 61 may be intermittent, which would indicate that it is initiated by processes other than radiative acceleration. Interestingly, our atmosphere models would confirm this hypothesis, because the force due to radiation pressure is much too low to explain the observed mass loss: For the massive WC star WR 111, which has a very similar spectral appearance to SMP 61, the radiation pressure, as calculated in our models, supplies about one half of the energy necessary to drive the stellar wind (Gräfener et al. 2002). On the other hand, for SMP 61 the same models provide only $17 \%$ of the wind energy. However, one must be aware that the timescales for mass-loss and evolution derived above both suffer from large uncertainties. The spectroscopically derived mass-loss rate depends on the clumping factor $\left(\dot{M} \propto 1 / \sqrt{D}\right.$ for constant $R_{\mathrm{t}}$, see Sect. 3.2) which is only roughly known. The error margin for $\dot{M}$ may therefore be as large as $\pm 50 \%$. The stellar evolutionary timescale is even more uncertain, because it depends on the stellar luminosity and the mass of the stellar envelope above the burning shell. The latter is strongly dependent on model assumptions. The nebular age is also uncertain, mostly because the nebular velocity changes during the course of evolution as shown by dynamical simulations (Mellema 1994; Villaver et al. 2002).

\section{Summary}

We have obtained HST STIS imaging and spectroscopic observations of the Magellanic Cloud planetary nebula SMP 61 and its central star. We have also searched the literature for other observational data concerning this object, in order to obtain a full set of observational constrains and to assess their uncertainties.

We have performed a detailed spectral analysis of the central star. For that, we used the Potsdam code for expanding atmospheres in non-LTE, which includes the statistical equations for many ions, including ions from the iron group. The observed stellar spectrum is well represented by a model with the following parameters: $L_{\star}=10^{3.96} L_{\odot}, R_{\star}=0.42 R_{\odot}, T_{\star}=$ $87.5 \mathrm{kK}, \dot{M}=10^{-6.12} M_{\odot} \mathrm{yr}^{-1}, v_{\infty}=1400 \mathrm{~km} \mathrm{~s}^{-1}$, clumping factor $D=4$ and $R_{\mathrm{t}}=4.63 R_{\odot}$. The abundances of the elements by mass are $X_{\mathrm{He}}=0.45, X_{\mathrm{C}}=0.52, X_{\mathrm{N}}<5 \times 10^{-5}, X_{\mathrm{O}}=0.03$,
$X_{\mathrm{Fe}}<1 \times 10^{-4}$. The low iron and nitrogen abundances indicate that the star has undergone an AGB final thermal pulse with a very low $\mathrm{H}$-envelope mass $\left(<9 \times 10^{-4} M_{\odot}\right)$. The iron depletion is probably due to s-process nucleosynthesis during previous thermal pulses.

The fluxes from the model stellar atmosphere were used as an input to our photoionization code to construct photoionization models of the nebula. We considered all the available observational constraints, within their error bars. These constraints are numerous and allow a detailed discussion of the models. We find that the fluxes from the stellar atmospheres allow to reproduce many features of the nebular emission. However, the observed nebular properties are better explained if the central star produces less photons in the Lyman continuum than the model atmosphere by about a factor 2. An even better fit is obtained if the Lyman continuum radiation is also softer than predicted by the stellar model. A possible solution involves the treatment of dielectronic recombination in the stellar atmosphere code and will be presented in a forthcoming paper.

We also find that the nebula must contain extremely carbon rich clumps in order to reproduce the observed C III] $\lambda 1909 / \mathrm{C}$ II $\lambda 4267$ ratio. The observational constraints do not allow us to describe these clumps in detail (density, chemical composition, location) but these clumps are likely produced by ejecta from the clumpy stellar wind, possibly partly mixed with the nebular material. Obviously, high signal-to noise and high spatial resolution observations of nearby planetary nebulae excited by a similar star would be extremely useful to better understand the process of enrichment of planetary nebulae by freshly made carbon (and helium).

From a comparison of the estimated total amount of carbon in the nebula with the carbon mass-loss rate measured in the central star, we infer that the strong WC mass-loss may have been active for only a limited period in the post-AGB evolution of SMP 61. However, this last result relies on uncertain timescales and is subject to revision.

Acknowledgements. This work has been partially supported by DGAPA/UNAM (grant 114601), CONACYT/México (grant 32594-E), by the CNRS-CONACYT joint project (number 10385) by the Deutsche Agentur für Raumfahrtangelegenheiten under grant DARA 50 OR 0008 and by the jumelage Astronomie France-Pologne.

\section{References}

Allende Prieto, C., Barklem, P. S., Asplund, M., \& Ruiz Cobo, B. 2001a, ApJ, 558, 830

Allende Prieto, C., Lambert, D. L., \& Asplund, M. 2001b, ApJ, 556, L63

Benedict, G. F., McArthur, B. E., Fredrick, L. W., et al. 2002, AJ, 124, 1695

Blöcker, T. 1995, A\&A, 299, 755

Blöcker, T. 2001, Ap\&SS, 275, 1

Clegg, R. E. S., Harrington, J. P., Barlow, M. J., \& Walsh, J. R. 1987, ApJ, 314, 551

Cohen, M. 2001, Ap\&SS, 275, 103 
Crowther, P. A., Abbott, J. B., Hillier, D. J., \& De Marco, O. 2003, in Planetary nebulae: Their Evolution and Role in the Universe, ed. M. Dopita, \& S. Kwok (San Francisco: ASP), IAU Symp., 209, in press

Crowther, P. A., Dessart, L., Hillier, D. J., Abbott, J. B., \& Fullerton, A. W. 2002, A\&A, 392, 653

Crowther, P. A., Pasquali, A., de Marco, O., et al. 1999, A\&A, 350, 1007

De Marco, O., \& Barlow, M. J. 2001, Ap\&SS, 275, 53

De Marco, O., \& Crowther, P. A. 1998, MNRAS, 296, 419

De Marco, O., \& Crowther, P. A. 1999, MNRAS, 306, 931

De Marco, O., Crowther, P. A., Barlow, M. J., Clayton, G. C., \& de Koter, A. 2001, MNRAS, 328, 527

De Marco, O., \& Soker, N. 2002, PASP, 114, 602

Dudziak, G., Péquignot, D., Zijlstra, A. A., \& Walsh, J. R. 2000, A\&A, 363, 717

Esteban, C. 2002, Rev. Mex. A\&A, 12, 56

Górny, S. K., \& Tylenda, R. 2000, A\&A, 362, 1008

Gräfener, G., Koesterke, L., \& Hamann, W.-R. 2002, A\&A, 387, 244

Gray, D. F. 1992, The observation and analysis of stellar photospheres (Cambridge University Press)

Grevesse, N., \& Sauval, A. J. 1998, Space Sci. Rev., 85, 161

Hamann, W.-R. 1985, A\&A, 148, 364

Hamann, W.-R. 1986, A\&A, 160, 347

Hamann, W.-R. 1997, in Planetary Nebulae, ed. H. J. Habing, \& H. J. G. L. M. Lamers (Kluwer, Dordrecht), IAU Symp., 180, 91

Hamann, W.-R., \& Gräfener, G. 2003, A\&A, submitted

Hamann, W.-R., \& Koesterke, L. 1998, A\&A, 335, 1003

Hamann, W.-R., Leuenhagen, U., Koesterke, L., \& Wessolowski, U. 1992, A\&A, 255, 200

Harrington, J. P., \& Feibelman, W. A. 1984, ApJ, 277, 716

Herwig, F. 2001a, Ap\&SS, 275, 15

Herwig, F. 2001b, ApJ, 554, L71

Herwig, F., Lugaro, M., \& Werner, K. 2003, in Planetary nebulae: Their Evolution and Role in the Universe, ed. M. Dopita, \& S. Kwok (San Francisco: ASP), IAU Symp., 209, in press

Hillier, D. J., \& Miller, D. L. 1999, ApJ, 519, 354

Jacoby, G. H., \& Ford, H. C. 1983, ApJ, 266, 298

Kingsburgh, R. L., \& Barlow, M. J. 1994, MNRAS, 271, 257

Koesterke, L. 2001, Ap\&SS, 275, 41

Koesterke, L., \& Hamann, W.-R. 1995, A\&A, 299, 503

Koesterke, L., \& Hamann, W.-R. 1997a, in Planetary Nebulae, IAU Symp., 180, 114

Koesterke, L., \& Hamann, W.-R. 1997b, A\&A, 320, 91

Koesterke, L., Hamann, W.-R., \& Gräfener, G. 2002, A\&A, 384, 562

Koesterke, L., Hamann, W.-R., \& Kosmol, P. 1992, A\&A, 255, 490

Leuenhagen, U., \& Hamann, W.-R. 1994, A\&A, 283, 567

Leuenhagen, U., Hamann, W.-R., \& Jeffery, C. S. 1996, A\&A, 312, 167

Li, J., Harrington, J. P., \& Borkowski, K. J. 2002, AJ, 123, 2676
Liu, X.-W, 2002, Rev. Mex. A\&A, 12, 70

Liu, X.-W., Storey, P. J., Barlow, M. J., et al. 2000, MNRAS, 312, 585

Lucy, L. B. 1964, in First Harvard-Smithsonian Conference on Stellar Atmospheres, ed. E. H. Avrett, O. J. Gingerich, \& C. A. Whitney, Smithsonian Astrophysical Observatory Special Report No. 167 (Cambridge, Mass.), 93

Lugaro, M., Herwig, F., Lattanzio, J. C., Gallino, R., \& Straniero, O. 2003, ApJ, 586, 1305

Meatheringham, S. J., \& Dopita, M. A. 1991, ApJS, 75, 407

Mellema, G. 1994, A\&A, 290, 915

Mihalas, D. 1971, ApJ, 170, 541

Mihalas, D. 1978, Stellar Atmospheres, 2nd ed. (San Francisco: W. H. Freeman and Company)

Miksa, S., Deetjen, J. L., Dreizler, S., et al. 2002, A\&A, 389, 953

Monk, D. J., Barlow, M. J., \& Clegg, R. E. S. 1988, MNRAS, 234, 583

Peña, M., Ruiz, M. T., \& Torres-Peimbert, S. 1997a, A\&A, 324, 674

Peña, M., Hamann, W.-R., Koesterke, L., et al. 1997b, ApJ, 491, 233

Peña, M., Stasińska, G., Esteban, C., et al. 1998, A\&A, 337, 866

Peña, M., Stasińska, G., \& Medina, S. 2001, A\&A, 367, 983

Rauch, T., Köppen, J., \& Werner, K. 1994, A\&A, 286, 543

Rauch, T., Köppen, J., \& Werner, K. 1996, A\&A, 310, 613

Schmutz, W., Hamann, W.-R., \& Wessolowski, U. 1989, A\&A, 210, 236

Seaton, M. J. 1979, MNRAS, 187, 73P

Stasińska, G., \& Leitherer, C. 1996, ApJS, 107, 661

Stasińska, G. 2002 [astro-ph/0207500]

Stasińska, G., \& Szczerba, R. 1999, A\&A, 352, 297

Stasińska, G., \& Szczerba, R. 2001, A\&A, 379, 1024

Szczerba, R., Górny, S. K., Stasińska, G., Siódmiak, N., \& Tylenda, R. 2001, Ap\&SS, 275, 113

Torres-Peimbert, S., Peimbert, M., \& Peña, M. 1990, A\&A, 233, 540

Tylenda, R., Acker, A., \& Stenholm, B. 1993, A\&AS, 102, 595

Unsöld, A. 1955, Physik der Sternatmosphären, 2nd ed. (Berlin: Springer Verlag)

Vassiliadis, E., Dopita, M. A., Bohlin, R. C., et al. 1998a, ApJS, 114, 237

Vassiliadis, E., Dopita, M. A., Meatheringham, S. J., et al. 1998b, ApJ, 503,253

Vassiliadis, E., \& Wood, P. R. 1994, ApJS, 92, 125

Villaver, E., Manchado, A., \& Garcia-Segura, G. 2002, ApJ, 581, 1204

Werner, K., Dreizler, S., Koesterke, L., \& Kruk, J. W. 2003, in Planetary Nebulae: Their Evolution and Role in the Universe, ed. M. Dopita, \& S. Kwok (San Francisco: ASP), IAU Symp., 209, in press

Wesson, R., Liu, X.-W., \& Barlow, M. J. 2003, MNRAS, 340, 253

Zijlstra, A. A., van Hoof, P. A. M., Chapman, M., \& Loup, C. 1994, A\&A, 290, 228 
G. Stasińska et al.: The planetary nebula LMC-SMP 61 and its [WC]-type central star, Online Material p 1

\section{Online Material}


G. Stasińska et al.: The planetary nebula LMC-SMP 61 and its [WC]-type central star, Online Material p 2

Table 2. Nebular dereddened fluxes from different authors.

\begin{tabular}{|c|c|c|c|c|c|c|}
\hline \multirow[t]{2}{*}{ lambda } & \multirow[t]{2}{*}{ ion } & \multicolumn{5}{|c|}{ Dereddened fluxes relative to $\mathrm{H} \beta$} \\
\hline & & STIS (this work) & PRT-P97a & MD91 & Vass98a & MBC88 \\
\hline 1240 & $\mathrm{NV}$ & stell & $\ldots$ & & $\ldots$ & \\
\hline 1336 & C II & $\ldots$ & $\ldots$ & & 26.0 & \\
\hline 1406 & Si IV], O IV] & stell & $\ldots$ & & 12.9 & \\
\hline 1550 & C IV & stell & stell & & $72.1+50.4$ & \\
\hline 1640 & He II & stell & $\ldots$ & & 31.2 & \\
\hline 1649 & C IV & stell & $\ldots$ & & 10.3 & \\
\hline 1666 & [O III] & 6.8: & $\ldots$ & & 7.4 & \\
\hline 1750 & $\mathrm{~N}$ III] & 0.09 & & & & \\
\hline 1909 & $\mathrm{C}$ III] & 316 & 401 & & 392.7 & \\
\hline 2297 & C III & stell & $\ldots$ & & 30.7 & \\
\hline 2321,26 & C II & 62 & $\ldots$ & & 87.9 & \\
\hline 2405 & C IV & stell & $\ldots$ & & 8.1 & \\
\hline 2470 & {$[\mathrm{O} \mathrm{II}]$} & 6.2 : & $\ldots$ & & 9.0 & \\
\hline 2524 & C IV & stell & $\ldots$ & & 11.9 & \\
\hline 3203 & He I,He II & stell & $\ldots$ & & 3.4 & \\
\hline $3726+29$ & [O II $]$ & 60 & 80.0 & 85.8 & & $<80$ \\
\hline 3835 & $\mathrm{H} 9$ & 7.2 & 7.4 & 10.4 & & $\ldots$ \\
\hline 3869 & {$[\mathrm{Ne}$ III] } & 43 & 41.6 & 50.6 & & 47.2 \\
\hline 3889 & $\mathrm{He} \mathrm{I}+\mathrm{H} 8$ & 19 & 18.7 & 24.6 & & $\ldots$ \\
\hline $3967+70$ & {$[\mathrm{Ne}$ III $]+\mathrm{H} 7$} & 28 & 29.0 & 37.9 & & $\ldots$ \\
\hline 4026 & $\mathrm{He} \mathrm{I}+\mathrm{He} \mathrm{II}$ & 2.6 & 2.5 & 3.9 & & $\ldots$ \\
\hline 4069 & {$[\mathrm{~S}$ II] } & 2.2 & 2.9 & 3.9 & & $\ldots$ \\
\hline 4102 & $\mathrm{H} \delta$ & 26 & 25.2 & 31.2 & & 13.5 \\
\hline 4269 & C II & 1.8 & 1.4 & 1.7 & & $\ldots$ \\
\hline 4340 & $\mathrm{H} \gamma$ & 46 & 46.6 & 46.6 & & 39.9 \\
\hline 4363 & [O III] & 5.3 & 5.7 & 6.4 & & 5.5 \\
\hline 4471 & He I & 5.2 & 4.9 & 5.9 & & $\ldots$ \\
\hline 4686 & He II & stell & stell & $\ldots$ & & $\ldots$ \\
\hline $4711+13$ & {$[\mathrm{Ar} I V]+\mathrm{He} \mathrm{I}$} & $\ldots$ & 0.7 & 1.3 & & $\ldots$ \\
\hline 4711 & [Ar IV] & $\ldots$ & 0.2 : & & & \\
\hline 4740 & [Ar IV] & $\ldots$ & 0.2 : & $\ldots$ & & $\ldots$ \\
\hline 4861 & $\mathrm{H} \beta$ & 100.0 & 100.0 & 100.0 & & 100.0 \\
\hline 4921 & He I & 1.5 & 1.4 & 2.0 & & $\ldots$ \\
\hline 4959 & [O III] & 253 & 231 & 222.5 & & 278.6 \\
\hline 5007 & [O III] & sat? & sat & sat & & 800.0 \\
\hline 5200 & {$[\mathrm{~N} \mathrm{I}]$} & & 0.3: & $\ldots$ & & $\ldots$ \\
\hline 5411 & He II & & 0.4 & $\ldots$ & & $\ldots$ \\
\hline 5522 & [Cl III] & & 0.2 : & ... & & ... \\
\hline 5538 & [Cl III] & & 0.3: & $\ldots$ & & $\ldots$ \\
\hline 5755 & {$[\mathrm{~N} \mathrm{II}]$} & & 0.7 & 1.4 & & $\ldots$ \\
\hline 5808 & C IV & & stell & $\ldots$ & & $\ldots$ \\
\hline 5876 & $\mathrm{He} \mathrm{I}$ & & 16.0 & 18.8 & & 15.2 \\
\hline 6300 & [O I] & & 4.3 & 6.1 & & 3.3 \\
\hline 6312 & [S III] & & 1.2 & 1.3 & & $\ldots$ \\
\hline 6363 & [O I] & & 1.3 & 2.3 & & $\ldots$ \\
\hline 6548 & {$[\mathrm{~N}$ II $]$} & & 15.8 & 15.0 & & 7.7 \\
\hline 6563 & $\mathrm{H} \alpha$ & & 285.8 & 282.1 & & 282.4 \\
\hline 6584 & {$[\mathrm{~N}$ II $]$} & & 31.5 & 44.8 & & 22.3 \\
\hline 6678 & $\mathrm{He} \mathrm{I}+\mathrm{He}$ II & & 4.2 & 6.1 & & $\ldots$ \\
\hline 6717 & [S II] & & 2.1 & 2.2 & & $\ldots$ \\
\hline 6731 & [S II] & & 3.8 & 4.9 & & 5.3 \\
\hline 7065 & He I & & 8.2 & 13.7 & & $\ldots$ \\
\hline 7136 & [A III] & & 8.8 & 16.3 & & ... \\
\hline $7320+30$ & [O II] & & 14.3 & $\ldots$ & & $\ldots$ \\
\hline $\log F(\mathrm{H} \beta)$ & & -12.80 & -12.61 & $\ldots$ & & $\ldots$ \\
\hline$C(\mathrm{H} \beta)$ & & 0.12 & 0.18 & 0.19 & & $1.07^{1}$ \\
\hline slit (") & & $0.2 \times 52$ & $2 ; 10$ & $\ldots$ & & $3.6 \times 3.6$ \\
\hline
\end{tabular}

\footnotetext{
${ }^{1}$ Value affected by atmospheric dispersion.
} 